\title{
Stability Analysis of the Backward Euler Time Discretization for the Pin- Resolved Transport Transient Reactor Calculation
}

\author{
Ang Zhu \\ Yunlin Xu \\ Thomas Downar \\ Department of Nuclear Engineering \& Radiological Science \\ University of Michigan, Ann Arbor, 48105, USA \\ angzhu@umich.edu \\ yunlin@umich.edu \\ downar@umich.edu
}

\begin{abstract}
Three-dimensional, full core transport modeling with pin-resolved detail for reactor dynamic simulation is important for some multi-physics reactor applications. However, it can be computationally intensive due to the difficulty in maintaining accuracy while minimizing the number of time steps. A recently proposed Transient Multi-Level (TML) methodology overcomes this difficulty by use multi-level transient solvers to capture the physical phenomenal in different time domains and thus maximize the numerical accuracy and computational efficiency. One major problem with the TML method is the negative flux/precursor number density generated using large time steps for the MOC solver, which is due to the backward Euler discretization scheme. In this paper, the stability issue of backward Euler discretization is first investigated using the Point Kinetics Equations (PKEs), and the predicted maximum allowed time step for SPERT test 60 case is shown to be less than $10 \mathrm{~ms}$. To overcome this difficulty, linear and exponential transformations are investigated using the PKEs. The linear transformation is shown to increase the maximum time step by a factor of 2, and the exponential transformation is shown to increase the maximum time step by a factor of 5 , as well as provide unconditionally stability above a specified threshold. The two sets of transformations are then applied to TML scheme in the MPACT code, and the numerical results presented show good agreement for standard, linear transformed, and exponential transformed maximum time step between the EPKE model and the MPACT whole core transport solution for three different cases, including a pin cell case, a 3D SPERT assembly case and a row of assemblies ("striped assembly case") from the SPERT model. Finally, the successful whole transient execution of the stripe assembly case shows the ability of the exponential transformation method to use $10 \mathrm{~ms}$ and $20 \mathrm{~ms}$ time steps, which all failed using the standard method.
\end{abstract}

Key Words: Backward Euler, Stability, Transformation

\section{INTRODUCTION}

Three-dimensional, full core modeling with pin-resolved detail has become the state of art computational simulation for nuclear reactors. However, the computational intensiveness has become challenging, especially for time-dependent transient analysis since it is difficult to maintain accuracy when using large time steps to minimize the number of transport solutions. As a result, several innovative methods have been investigated to more efficiency solve the timedependent Boltzmann equation. One of most popular and widely used methods has been the quasi-static method which originated with Henry [1,2] in 1958 and continued with Ott and 
Meneley [3] who proposed the Improved Quasi-Static (IQS) method in 1969. The underlying premise of the quasi-static method is that the flux can be factorized into an amplitude and a shape function since the amplitude of the flux changes much more rapidly than the flux shape. In the IQS method the shape function is solved by a modified time-dependent Boltzmann equation with a known amplitude function, and the amplitude function is calculated using the PKEs which are formed by integrating the time-dependent Boltzmann equation with the known shape function. The shape and amplitudes functions are iteratively solved with a shape constraint function until the convergence is satisfied. Recently, Ban [4] adopted the nodal/PKEs based IQS method and innovated a new fine/coarse mesh based IQS method by introducing a coarse mesh wise rather than whole core amplitude function, which was named Multigrid Amplitude Function (MAF) and was later implemented in a transport based transient solver [5, 6].

In addition to the IQS/MAF method, a new factorization method called Predictor-Corrector Quasi-Static method (PCQM) [7] has recently become popular. Instead of solving the shape and amplitude functions, the PCQM computes the flux directly in the predictor step and the flux is corrected using the amplitude function evaluated at the corrector step. Several studies $[8,9$, and 10] have applied the PCQM to the nodal/PKE scheme and have shown improved accuracy and computational efficiency over traditional IQS method by avoiding the iteration between shape and amplitude function in the PCQM method.

Recently, a Transient Multi-Level (TML) method was proposed by Zhu [11] and the PCQM iteration scheme is used for coupling of both the 3D-Transport/3D-CMFD level and the 3DCMFD/PKEs level. At each level, the original flux equation is solved in the coarse predictor step and then is factorized as an amplitude and a shape function in the corrector step, where the predicted solution is corrected using multiple fine steps. In the first level 3D-Transort/3D-CMFD coupling, the angular and sub-pin flux shape function in the Boltzmann transport equation are assumed to vary slowly over time and the CMFD cell wise amplitude function is solved using multiple steps of the CMFD transient equation. In the second level, the CMFD scalar flux calculated in the last step is further corrected by a whole core amplitude function generated by solution of the Point Kinetics Equations (PKE). The numerical results in [11] show a considerable reduction of the computational time can be achieved while maintaining a high level of numerical accuracy.

However, one major challenge of the TML method is that the flux/precursor number density generated by the transport step can be negative when using large time steps in the backward Euler discretization. This imposes significant limits on the performance of the TML method and is the focus of the research described in this paper. The stability issue is first investigated using PKEs and found to be about 10ms for typical reactivity insertion accidents (RIA), such as the SPERT (Special Power Excursion Reactor Test) test 60 [12]. Two transformation methods are proposed in this paper to overcome this problem, including both linear and exponential transformation methods. The exponential transformation is a legacy method [13, 14 and 15], which has successfully accelerated the diffusion type transient problem. However, as discussed 
in [4], the method has not improved the accuracy of the transient kinetics calculation for their applications.

The paper is organized as follows. Section 2 provides a detailed description of the transient solvers developed in MPACT [16], including both the 3D-transport and the PKEs transient formulations. In section 3, the stability issue for backward Euler discretization with large time step is investigated using PKEs models. Section 4 is devoted to the two transient transformation methods and their stability analysis using PKEs model. Section 5 then describes the application of linear and exponential transformations to the MPACT transport transient solver. Finally, Section 6 provides numerical stability results using the PKEs and MPACT for the standard, linear and exponential transformation methods, using a pin cell, a 3D SPERT [17] assembly and a row of SPERT assemblies in a "stripe" model.

\section{TRANSIENT METHODOLOGY}

The transient capability was recently added to the MPACT [18] code and the detail formulation of the equations for the transient solvers are presented in this section, including the 3D-transport transient equations and the PKEs.

\subsection{D-Transport Transient Equation}

The 3D transport transient solver begins with the continuous 3D Boltzmann equation and the neutron precursor equations:

$$
\begin{aligned}
& \frac{1}{v(E)} \frac{\partial \varphi(\mathbf{r}, \boldsymbol{\Omega}, E, t)}{\partial t}=-\boldsymbol{\Omega} \cdot \nabla \varphi(\mathbf{r}, \boldsymbol{\Omega}, E, t)-\Sigma_{t}(r, E, t) \varphi(\mathbf{r}, \boldsymbol{\Omega}, E, t) \\
& \quad+\int_{0}^{\infty} \int_{0}^{4 \pi} \Sigma_{s}\left(\mathbf{r}, \boldsymbol{\Omega} \cdot \boldsymbol{\Omega}^{\prime}, E^{\prime} \rightarrow E, t\right) \varphi\left(\mathbf{r}, \boldsymbol{\Omega}^{\prime}, E^{\prime}, t\right) d \Omega^{\prime} d E^{\prime} \\
& \quad+\frac{1}{4 \pi}\left(\chi_{p}(\mathbf{r}, E, t)(1-\beta(\mathbf{r}, t)) S_{F}(\mathbf{r}, t)+\chi_{d}(\mathbf{r}, E, t) S_{d}(\mathbf{r}, t)\right) \\
& \frac{d C_{k}(\mathbf{r}, t)}{d t}=\beta_{k}(\mathbf{r}, t) S_{F}(\mathbf{r}, t)-\lambda_{k}(\mathbf{r}, t) C_{k}(\mathbf{r}, t), k=1,2, \ldots, 6
\end{aligned}
$$

Where $\varphi$ and $C_{k}$ are the angular flux and the delayed neutron precursor density, and $S_{F}$ and $S_{d}$ are the total fission source and the delayed neutron source which are defined as:

$$
S_{F}(\mathbf{r}, t)=\frac{1}{k_{e f f}^{s}} \int_{0}^{\infty} v \Sigma_{f}\left(\mathbf{r}, E^{\prime}, t\right) \phi\left(\mathbf{r}, E^{\prime}, t\right) d E^{\prime}
$$




$$
S_{d}(\mathbf{r}, t)=\sum_{k=1}^{6} \lambda_{k}(\mathbf{r}, t) C_{k}(\mathbf{r}, t)
$$

In the above equations, the $S_{F}$ value is adjusted by the eigenvalue determined in the steady-state calculation used to initialize the transient.

For a given time step size $\Delta t_{n}$ at time step $n$, Eq. (1) can be discretized using the backward Euler method as:

$$
\frac{\varphi^{n}(\mathbf{r}, \boldsymbol{\Omega}, E)-\varphi^{n-1}(\mathbf{r}, \boldsymbol{\Omega}, E)}{v(E) \Delta t_{n}}=R^{n}(\mathbf{r}, \boldsymbol{\Omega}, E)
$$

with $R^{n}$ denoting all the right hand side terms of Eq. (1) at time step $n$.

The Boltzmann equation is coupled to the precursor equations by integrating Eq.(2) and the precursor equations with a second order approximation for the fission source is written as in Eq. (6):

$$
C_{k}^{n}(\mathbf{r})=\Omega_{k}^{0}\left(\tilde{\lambda}_{k}^{n}\right) C_{k}^{n-1}(\mathbf{r})+\frac{1}{\lambda_{k}^{n}}\left[\beta_{k}^{n}(\mathbf{r}) S_{F}^{n}(\mathbf{r}) \Omega_{k}^{n}\left(\tilde{\lambda}_{k}^{n}\right)+\beta_{k}^{n-1}(\mathbf{r}) S_{F}^{n-1}(\mathbf{r}) \Omega_{k}^{n-1}\left(\tilde{\lambda}_{k}^{n}\right)+\beta_{k}^{n-2}(\mathbf{r}) S_{F}^{n-2}(\mathbf{r}) \Omega_{k}^{n-2}\left(\tilde{\lambda}_{k}^{n}\right)\right]
$$

where $\tilde{\lambda}_{k}^{n}=\lambda_{k}^{n} \Delta t_{n} E(x)=e^{-x}, \kappa_{0}(x)=1-e^{-x}, \kappa_{1}(x)=1-\frac{\kappa_{0}(x)}{x}, \kappa_{2}(x)=1-\frac{2 \kappa_{1}(x)}{x}$,

$$
\begin{aligned}
& \Omega_{k}^{0}\left(\tilde{\lambda}_{k}^{n}\right)=E\left(\tilde{\lambda}_{k}^{n}\right), \Omega_{k}^{n}\left(\tilde{\lambda}_{k}^{n}\right)=\frac{\kappa_{2}\left(\tilde{\lambda}_{k}^{n}\right)+\gamma \kappa_{1}\left(\tilde{\lambda}_{k}^{n}\right)}{(1+\gamma)} \\
& \Omega_{k}^{n-1}\left(\tilde{\lambda}_{k}^{n}\right)=\left(\kappa_{0}\left(\tilde{\lambda}_{k}^{n}\right)-\frac{\kappa_{2}\left(\tilde{\lambda}_{k}^{n}\right)+(\gamma-1) \kappa_{1}\left(\tilde{\lambda}_{k}^{n}\right)}{\gamma}\right) \\
& \Omega_{k}^{n-2}\left(\tilde{\lambda}_{k}^{n}\right)=\frac{\kappa_{2}\left(\tilde{\lambda}_{k}^{n}\right)-\kappa_{1}\left(\tilde{\lambda}_{k}^{n}\right)}{(1+\gamma) \gamma}
\end{aligned}
$$

Thus, the delayed neutron source can be expressed as:

$$
S_{d}^{n}(\mathbf{r})=\omega^{n}(\mathbf{r}) S_{F}^{n}(\mathbf{r})+\tilde{S}_{d}^{n-1}(\mathbf{r})
$$

Where $\omega^{n}=\sum_{k=1}^{6} \beta_{k} \Omega_{k}^{n}\left(\tilde{\lambda}_{k}^{n}\right)$ and

$$
\tilde{S}_{d}^{n-1}(\mathbf{r})=\sum_{k=1}^{6} \lambda_{k} \Omega_{k}^{0}\left(\tilde{\lambda}_{k}^{n}\right) C_{k}^{n-1}(\mathbf{r})+S_{F}^{n-1}(\mathbf{r}) \sum_{k=1}^{6} \beta_{k}^{n-1}(\mathbf{r}) \Omega_{k}^{n-1}\left(\tilde{\lambda}_{k}^{n}\right)+S_{F}^{n-2}(\mathbf{r}) \sum_{k=1}^{6} \beta_{k}^{n-2}(\mathbf{r}) \Omega_{k}^{n-2}\left(\tilde{\lambda}_{k}^{n}\right)
$$


By inserting the delayed neutron source terms Eq. (8) into Eq. (1) and expressing all the terms of $R^{n}$ explicitly, and applying an isotropic assumption to $\partial \varphi / \partial t$, the final solution can be simplified as:

$$
\begin{aligned}
& \mathbf{\Omega} \nabla \varphi^{n}(\mathbf{r}, \mathbf{\Omega}, E)+\sum_{t}^{n} \varphi^{n}(\mathbf{r}, \boldsymbol{\Omega}, E)=\int_{0}^{\infty} \int_{0}^{4 \pi} \Sigma_{s}^{n}\left(\mathbf{r}, \mathbf{\Omega} \cdot \boldsymbol{\Omega}^{\prime}, E^{\prime} \rightarrow E\right) \varphi^{n}\left(\mathbf{r}, \mathbf{\Omega}^{\prime}, E^{\prime}\right) d \Omega^{\prime} d E^{\prime} \\
& +\frac{\chi^{n}(\mathbf{r}, E) S_{F}^{n}(\mathbf{r})}{4 \pi}+\frac{S_{t r}^{n}(\mathbf{r}, E)}{4 \pi}
\end{aligned}
$$

Where the transient source is defined in Eq. (10) by using the correlation $\chi(\mathbf{r}, E)=\chi_{p}(\mathbf{r}, E)(1-\beta(\mathbf{r}))+\chi_{d}(\mathbf{r}, E) \beta(\mathbf{r})$ :

$$
S_{t r}^{n}(\mathbf{r}, E)=A(\mathbf{r}, E) \phi_{g}^{n}+B(\mathbf{r}, E) S_{F}^{n}+C(\mathbf{r}, E)
$$

where $A$ and $B$ are flux and fission source dependent coefficients, while $C$ is a constant coefficient:

$$
\begin{aligned}
& A(\mathbf{r}, E)=-\frac{1}{v(E) \Delta t_{n}}, B(\mathbf{r}, E)=\chi_{d}(\mathbf{r}, E)\left(\omega^{n}(\mathbf{r})-\beta(\mathbf{r})\right) \\
& C(\mathbf{r}, E)=\chi_{d}(\mathbf{r}, E) \tilde{S}_{d}^{n-1}(\mathbf{r}, E)+\frac{\phi^{n-1}(\mathbf{r}, E)}{v(E) \Delta t_{n}}
\end{aligned}
$$

Eq. (9) can be solved using any standard steady-state neutron transport solver by discretizing over space, angle, and energy, and applying a transient source term. In MPACT [18], a 2D-1D approach is used with 2D radial MOC and 1D axial NEM (Nodal Expansion Method) coupled with transverse leakage terms. In addition, a multi-group CMFD (Coarse Mesh Finite Difference) method has been successfully applied to accelerate the convergence of the solution of the transport equation, where the derivation can be found in [11].

\subsection{Point Kinetics Equations (PKEs)}

The PKEs are derived by integrating the CMFD equations using the adjoint flux [20]. The detail derivation is provided in several references [8,9 and 19] and the PKEs can be written as:

$$
\frac{d p(t)}{d t}=\frac{\rho(t)-\beta^{e f f}(t)}{\Lambda(t)} p(t)+\frac{1}{\Lambda(0)} \sum_{k} \lambda_{k}(t) \zeta_{k}(t)
$$




$$
\frac{d \zeta_{k}(t)}{d t}=\frac{\Lambda(0)}{\Lambda(t)} \beta_{k}^{e f f}(t) p(t)-\lambda_{k}^{P K}(t) \zeta_{k}(t), k=1,2 \ldots, 6
$$

where $p(t)$ represents the core wise amplitude function and $\zeta_{k}(t)$ is the adjoint flux weighted precursor number density for delayed group $k$.

The reactivity, delayed neutron fractions, neutron generation time, and delayed neutron constants are defined in Eq. (14) to Eq. (17) respectively:

$$
\begin{gathered}
\rho(t)=\frac{\left\langle\phi^{*}(\mathbf{r}, E)(\mathbf{F}-\mathbf{M}) \phi(\mathbf{r}, E, t)>\right.}{F(t)} \\
\beta_{k}^{e f f}(t)=\frac{<\phi^{*}(\mathbf{r}, E) \chi_{d k}(\mathbf{r}, E) \beta_{k}(\mathbf{r}) S^{F}(\mathbf{r}, t)>}{F(t)}, k=1,2 \ldots, 6 \quad \beta^{e f f}(t)=\sum_{k} \beta_{k}^{e f f}(t) \\
\Lambda(t)=\frac{<\phi^{*}(\mathbf{r}, E) \frac{1}{v(E)} \phi(\mathbf{r}, E, t)>}{F(t)} \\
\lambda_{k}^{P K}(t)=\frac{<\phi^{*}(\mathbf{r}, E) \lambda_{k}(\mathbf{r}, t) \chi_{d k}(\mathbf{r}, E) C_{k}(\mathbf{r}, t)>}{<\phi^{*}(\mathbf{r}, E) \chi_{d k}(\mathbf{r}, E) C_{k}(\mathbf{r}, t)>}
\end{gathered}
$$

where $F(t)=<\phi^{*}(\mathbf{r}, E) \chi(\mathbf{r}, E) S^{F}(\mathbf{r}, t)>$

Eq. (12) and Eq. (13) are solved using the same discretization applied to the transport transient equation, including backward Euler discretization for the $d p(t) / d t$ term and a second order approximation for the amplitude function during a time step $n$. The corresponding precursor solution to Eq. (13) is:

$$
\zeta_{k}^{n}=\Omega_{k, p k}^{0} \zeta_{k}^{n-1}+\frac{\Lambda(0)}{\lambda_{k}^{P K}}\left[p^{n} \frac{\beta_{k}^{e f f, n}}{\Lambda^{n}} \Omega_{k, p k}^{n}+p^{n-1} \frac{\beta_{k}^{e f f, n-1}}{\Lambda^{n-1}} \Omega_{k, p k}^{n-1}+p^{n-2} \frac{\beta_{k}^{e f f, n-2}}{\Lambda^{n-2}} \Omega_{k, p k}^{n-2}\right]
$$


where $\Omega_{k, p k}^{i}$ denotes the corresponding $\Omega_{k}^{i}$ parameters with the new effective delayed constant defined in Eq. (7) rather than region wise delayed constants. By applying the backward Euler discretization $d p(t) / d t$ and inserting Eq. (18) into Eq. (12), the current step power can be solved and the precursor equation is then calculated by inserting the power back into Eq. (18).

\section{NUMERICAL STABILITY OF THE TRANSIENT CALCULATION}

\subsection{Stable region of Backward Euler Time Discretization}

The analysis for the Backward Euler stability starts with a standard ODE:

$$
\frac{d y}{d t}=\lambda y
$$

The backward Euler discretization is:

$$
\frac{y^{n}-y^{n-1}}{\Delta t}=\lambda y^{n}
$$

The corresponding solution at time step $n$ is:

$$
y^{n}=\frac{1}{1-\lambda \Delta t} y^{n-1}
$$

For reactor transient analysis, all the flux/precursor number densities are positive, and the following Eq. (22) has to be satisfy to maintain the positivity of the next time step solution for all given positive eigenvalues.

$$
\Delta t^{\max }<\frac{1}{\lambda_{\text {positive }}^{\max }}
$$

A simple numerical result for backward Euler discretization is presented in Figure 1, where the $\lambda$ in Eq. (19) is set to be 50 and thus the critical time step is $20 \mathrm{~ms}$. The reference solution is generated by directly integrating Eq. (19) and the initial $y$ value is set to be unity. As illustrated, the solution using backward Euler becomes infinite around $20 \mathrm{~ms}$ and negative beyond $20 \mathrm{~ms}$, which is the source of instability of this method. For accuracy purpose (can be adjusted by predictor-corrector method), the time step used should be at least smaller than $75 \%$ of critical time step, which is around 15ms shown in Figure 1. 




Figure 1 the solution using reference and backward Euler.

\subsection{Eigenvalues of 6-group Point Kinetics Equation}

To estimate the maximum eigenvalue of a realistic operating reactor, the simplified PKEs in Eq. (12) and Eq. (13) are used. To simplify the derivation, the backward Euler discretization rather than second order integration method is applied to the precursor equations.

Assume the solution of $p(t)$ and $\zeta_{k}$ are in the form exponential form:

$$
P(t)=C_{2} \exp \left(\alpha_{n} t\right), \zeta_{k}(t)=C_{1, k} \exp \left(\alpha_{n} t\right), k=1,2 \ldots, 6
$$

Then the Eq. (12) and Eq. (13) can be written as:

$$
\begin{aligned}
\alpha_{n} \zeta_{k}(t) & =\frac{\Lambda(0)}{\Lambda(t)} \beta_{k}^{\text {eff }}(t) p(t)-\lambda_{k}^{P K}(t) \zeta_{k}(t), k=1,2 \ldots, 6 \\
\alpha_{n} p(t) & =\frac{\rho(t)-\beta^{e f f}(t)}{\Lambda(t)} p(t)+\frac{1}{\Lambda(0)} \sum_{k} \lambda_{k}(t) \zeta_{k}(t)
\end{aligned}
$$

By assuming constant dynamic parameters in one time step, the above equations can be written in a matrix form in Eq. (26), which is a standard eigenvalue problem and the numerical eigenvalues can be easily calculated. 


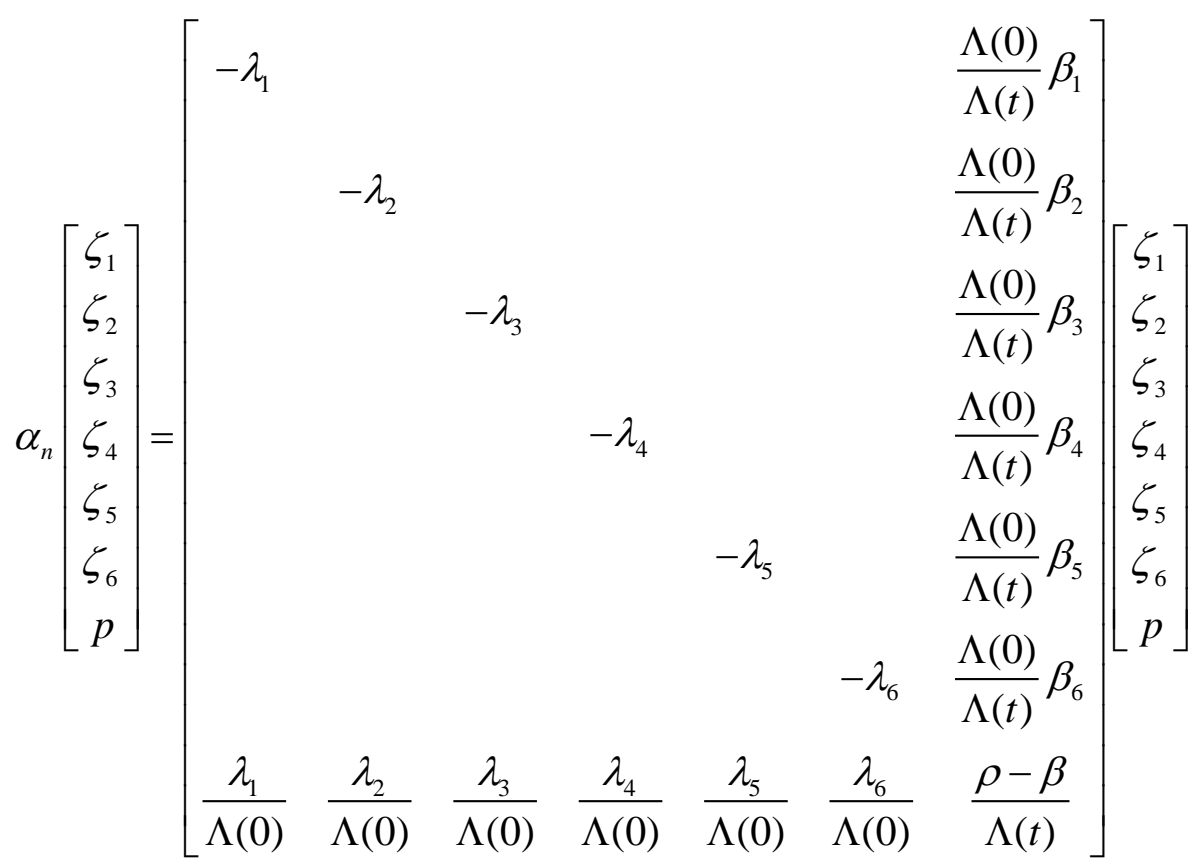

\subsection{Analytical Eigenvalues of 1-group Point Kinetics Equation}

To obtain insight on the dependence of the maximum eigenvalue as a function of the dynamic parameters, the delay neutrons are collapsed to one group and the corresponding PKEs in matrix form then becomes:

$$
\frac{d}{d t}\left(\begin{array}{l}
p \\
\zeta
\end{array}\right)=\left(\begin{array}{cc}
\frac{\rho-\beta^{e f f}}{\Lambda} & \frac{\lambda}{\Lambda_{0}} \\
\beta^{e f f} & -\lambda
\end{array}\right)\left(\begin{array}{l}
p \\
\zeta
\end{array}\right)
$$

The maximum eigenvalue of the above equation can be calculated directly, which is identical from the maximum time step from the Fourier analysis in [21]:

$$
\Delta t_{\text {standard }}^{\max }=\frac{2}{\left(\frac{\rho-\beta^{\text {eff }}}{\Lambda}-\lambda\right)+\sqrt{\left(\frac{\rho-\beta^{e f f}}{\Lambda}+\lambda\right)^{2}+4 \frac{\lambda}{\Lambda_{0}} \beta_{k}^{e f f}}}
$$

If the inserted reactivity is large, the last term in the denominator is small compared to the other terms and can be dropped. Thus the above equation can be further simplified as shown in Eq. (29), which is also known as the prompt jump approximation [22].

$$
\Delta t_{\text {standard }}^{\max } \approx \frac{\Lambda}{\rho(t)-\beta^{e f f}}
$$


For the SPERT test 60 result [12], the maximum time step is around 10ms based on Eq. (29) $(\Lambda=1.6 E-5, \beta=7.7 E-3, \rho=\$ 1.2)$.

\subsection{Comparison of the Eigenvalue Prediction of the Three PK Models.}

Numerical comparison of these three PK models is shown in Figure 2, where the dynamic parameters are documented in Table 1 (with generation time 2.243E-05s).

Table 1 Group wise dynamic data for comparison of three models.

\begin{tabular}{lllllll}
\hline Group & 1 & 2 & 3 & 4 & 5 & 6 \\
\hline Decay constant & $1.34 \mathrm{E}-02$ & $3.27 \mathrm{E}-02$ & $1.21 \mathrm{E}-01$ & $3.04 \mathrm{E}-01$ & $8.52 \mathrm{E}-01$ & $2.86 \mathrm{E}+00$ \\
$\begin{array}{llllll}\text { Delayed Neutron } \\
\text { Fraction }\end{array}$ & & & & & & \\
& $2.36 \mathrm{E}-04$ & $1.25 \mathrm{E}-03$ & $1.21 \mathrm{E}-03$ & $2.79 \mathrm{E}-03$ & $1.22 \mathrm{E}-03$ & $5.10 \mathrm{E}-04$ \\
\hline
\end{tabular}

For a short time scale, the one group delay constant is generated by Eq. (30) based on [22]:

$$
\lambda=\frac{\sum_{i} \lambda_{i} \beta_{i}}{\sum_{i} \beta_{i}}
$$




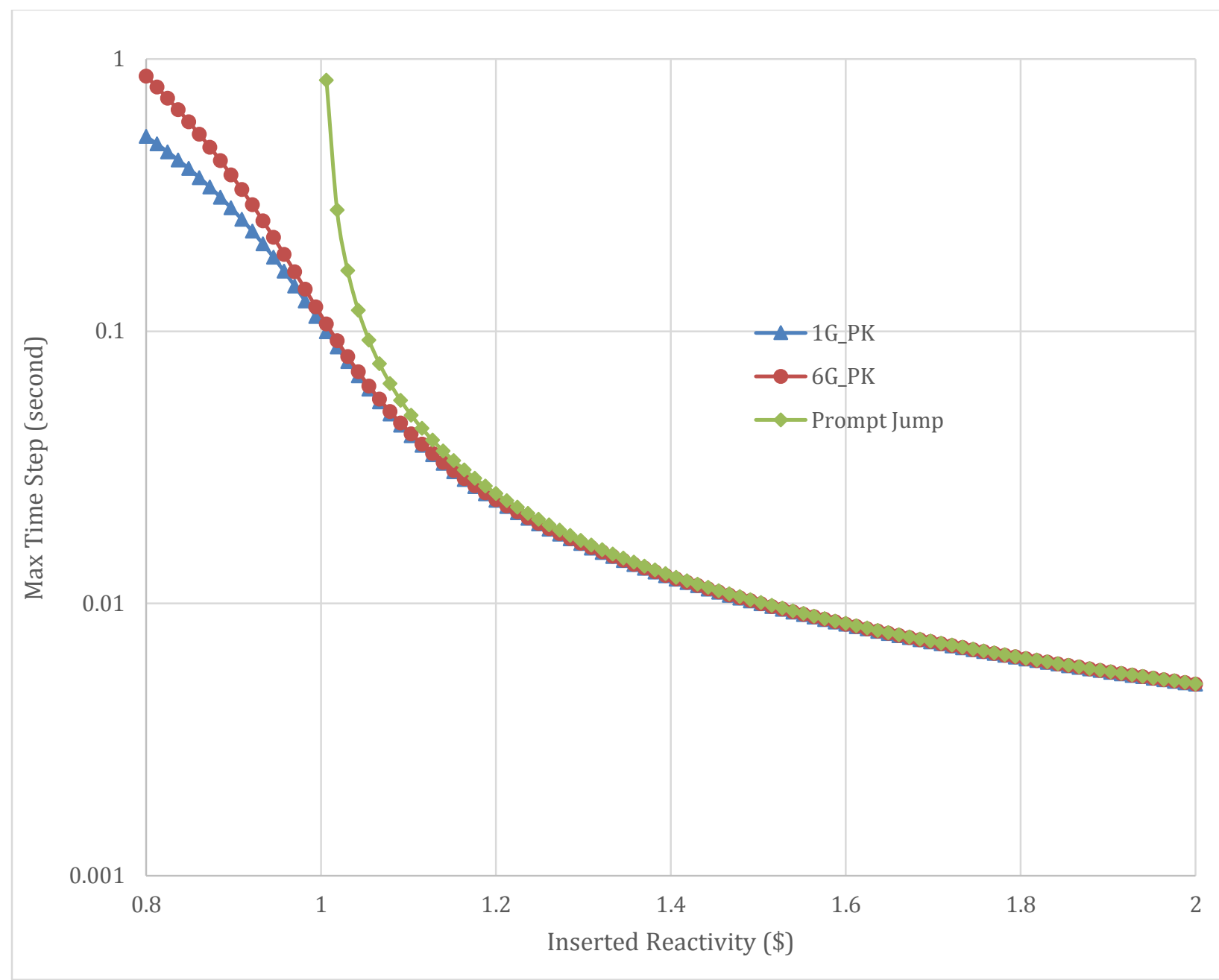

Figure 2 Maximum time step predicted by three point kinetics models

As indicated, when the reactivity insertion is large, similar behavior is observed for all three models. However, for a reactivity insertion smaller than $\$ 1.1$ the prompt jump model overestimates the maximum time step compared to the $6 \mathrm{G}$ model, and when the reactivity insertion is less than $\$ 1.0$, the $1 \mathrm{G}$ model underestimates the maximum time step compared to the $6 \mathrm{G}$ model. However, the $1 \mathrm{G}$ model in Eq. (28) provides an analytical lower bound for the maximum time step for the $6 \mathrm{G}$ model and therefore it will be used for the analysis in the remainder of the paper.

\section{AMPLITUDE TRANSFORMATION USING POINT KINETICS}

With the standard PKEs analysis from the previous section, the maximum allowed time step is bounded by Eq. (29), which is around $10 \mathrm{~ms}$ for a typical RIA condition. To address this limitation, linear and exponential transformations are introduced and their effectiveness is demonstrated using the point kinetics models. 


\section{Lemma 1}

$\frac{d}{d t} \mathbf{y}=\mathbf{A y}$ is stable using backward Euler method iff all the eigenvalues of matrix $\mathbf{I}-\mathbf{A} \Delta t$ are positive.

\section{Proof:}

If the backward Euler discretization for time is applied to $\frac{d}{d t} \mathbf{y}=\mathbf{A y}$, it becomes:

$$
\mathbf{y}^{n}=(\mathbf{I}-\mathbf{A} \Delta t)^{-1} \mathbf{y}^{n-1}
$$

Since all values in vector $\mathbf{y}^{n-1}$ are positive, the eigenvalues of $(\mathbf{I}-\mathbf{A} \Delta t)^{-1}$ must be positive in order to make $\mathbf{y}^{n}$ positive. It is easy to prove that the reciprocal of eigenvalue for a matrix is the eigenvalue for the inverse of the matrix, and thus all the eigenvalues of matrix $\mathbf{I}-\mathbf{A} \Delta t$ have to be positive.

\subsection{Linear transformation}

The linear transformation method can be most easily demonstrated by factorizing the power function of the point kinetics equation into a "shape" and amplitude function, where the amplitude function $A(t)$ is assumed to be "pre-calculated".

$$
p(t)=A(t) B(t)
$$

If the chain rule and linear approximation for the amplitude function illustrated in Eq. (33) is used, Eq. (12) becomes Eq. (34):

$$
\begin{gathered}
\frac{d A(t)}{d t}=\frac{A_{n}-A_{n-1}}{\Delta t} \\
\frac{1-A^{n-1} / A^{n}}{\Delta t} B(t)+\frac{d B(t)}{d t}=\frac{\rho(t)-\beta^{e f f}(t)}{\Lambda(t)} B(t)+\frac{1}{A^{n} \Lambda_{0}} \lambda(t) \zeta(t)
\end{gathered}
$$

Eq. (34) and the precursor equation Eq. (13) can be written into a matrix form:

$$
\frac{d}{d t}\left(\begin{array}{l}
B \\
\zeta
\end{array}\right)=\left(\begin{array}{cc}
\frac{\rho-\beta^{e f f}}{\Lambda}-\frac{1-A^{n-1} / A^{n}}{\Delta t} & \frac{\lambda}{A^{n} \Lambda_{0}} \\
\beta_{k}^{e f f} A^{n} & -\lambda
\end{array}\right)\left(\begin{array}{l}
B \\
\zeta
\end{array}\right)
$$


Then using backward Euler discretization of the left hand side of Eq. (35):

$$
\left.I-\Delta t\left(\begin{array}{ccc}
\frac{\rho-\beta^{e f f}}{\Lambda}-\frac{1-A^{n-1} / A^{n}}{\Delta t} & \frac{\lambda}{A^{n} \Lambda_{0}} \\
\beta_{k}^{\text {eff }} A^{n} & -\lambda
\end{array}\right)\right)\left(\begin{array}{l}
B^{n} \\
\zeta^{n}
\end{array}\right)=\left(\begin{array}{l}
B^{n-1} \\
\zeta^{n-1}
\end{array}\right)
$$

According to Lemma 1, all the eigenvalues of matrix in Eq. (36) should be nonnegative, which is identical to Eq. (37).

$$
\left(\frac{1+\alpha}{\Delta t}-\frac{\rho(t)-\beta^{e f f}(t)}{\Lambda(t)}\right)\left(\frac{1}{\Delta t}+\lambda\right)>\frac{\lambda}{\Lambda_{0}} \beta_{k}^{\text {eff }}(t)
$$

Where $1-A^{n-1} / A^{n}=\alpha, 0 \leq \alpha<1$

For problems that become numerically unstable (e.g. superprompt critical transients with reactivity inserted greater than total delay fraction), there is no $\alpha$ such that the linear transformation is unconditionally stable. This can be seen by setting $\Delta t$ in Eq. (37) to be infinite and the left hand side of the above equation is becomes negative which violates the inequality.

The maximum time step with linear transformation in Eq. (36) can be easily derived as:

$$
\Delta t_{\text {linear }}^{\max }=\frac{1}{\lambda_{\max }}=\frac{2}{\left(\frac{1}{1+\alpha} \frac{\rho-\beta^{\text {eff }}}{\Lambda}-\lambda\right)+\sqrt{\left(\frac{1}{1+\alpha} \frac{\rho-\beta^{\text {eff }}}{\Lambda}+\lambda\right)^{2}+4 \frac{\lambda}{\Lambda_{0}} \beta_{k}^{\text {eff }} \frac{1}{1+\alpha}}}
$$

If no linear transformation is performed and $\alpha$ is set to be unity, the above equation is the same as Eq. (28), which can be derived from the standard PKEs.

Since $\frac{\rho-\beta^{\text {eff }}}{\Lambda} \gg \lambda$ for most cases, it is expected that the maximum time step by the linear transformation would follow the " $1+\alpha$ " rule, which would predict a maximum of 2 . 


$$
\begin{aligned}
& \Delta t_{\text {linear }}^{\max } \approx \frac{2}{\left(\frac{1}{1+\alpha} \frac{\rho-\beta^{\text {eff }}}{\Lambda}-\frac{1}{1+\alpha} \lambda\right)+\sqrt{\left(\frac{1}{1+\alpha} \frac{\rho-\beta^{\text {eff }}}{\Lambda}+\frac{1}{1+\alpha} \lambda\right)^{2}+4 \frac{\lambda}{\Lambda_{0}} \beta_{k}^{\text {eff }} \frac{1}{1+\alpha}}} \\
& <\frac{2}{\left(\frac{1}{1+\alpha} \frac{\rho-\beta^{\text {eff }}}{\Lambda}-\frac{1}{1+\alpha} \lambda\right)+\sqrt{\left(\frac{1}{1+\alpha} \frac{\rho-\beta^{\text {eff }}}{\Lambda}+\frac{1}{1+\alpha} \lambda\right)^{2}+4 \frac{\lambda}{\Lambda_{0}} \beta_{k}^{\text {eff }}\left(\frac{1}{1+\alpha}\right)^{2}}} \\
& =(1+\alpha) \Delta t_{\text {standard }}^{\max }<2 \Delta t_{\text {stan dard }}^{\max }
\end{aligned}
$$

However, with the restriction in Eq. (22), the $1+\alpha$ value cannot exceed $2-1 / e^{1}=1.63$ in one time step, which makes the increase for the maximum time step from the linear transformation to be less than $63 \%$.

\subsection{Exponential transformation}

Since the linear transformation can only increase the maximum time step by a factor of 1.63 , a second transformation was investigated which is widely known as the exponential transformation:

$$
A(t)=A_{0} e^{\gamma t}
$$

The corresponding matrix form is then:

$$
\frac{d}{d t}\left(\begin{array}{l}
B \\
\zeta
\end{array}\right)=\left(\begin{array}{cc}
\frac{\rho(t)-\beta^{e f f}}{\Lambda}-\gamma & \frac{\lambda}{A_{0} e^{\gamma t} \Lambda_{0}} \\
\frac{\Lambda_{0}}{\Lambda} \beta_{k}^{e f f} A_{0} e^{\gamma t} & -\lambda
\end{array}\right)\left(\begin{array}{l}
B \\
\zeta
\end{array}\right)
$$

With the backward Euler discretization, Eq. (41) becomes:

$$
\left(I-\Delta t\left(\begin{array}{cc}
\frac{\rho-\beta^{e f f}}{\Lambda}-\gamma & \frac{\lambda}{A_{0} e^{\gamma t} \Lambda_{0}} \\
\frac{\Lambda_{0}}{\Lambda} \beta_{k}^{e f f} A_{0} e^{\gamma t} & -\lambda
\end{array}\right)\right)\left(\begin{array}{c}
B^{n} \\
\zeta^{n}
\end{array}\right)=\left(\begin{array}{c}
B^{n-1} \\
\zeta^{n-1}
\end{array}\right)
$$

To maintain the positivity of the eigenvalues, the following inequality should be satisfied: 


$$
\left(\frac{1}{\Delta t}-\frac{\rho-\beta^{e f f}}{\Lambda}+\gamma\right)\left(\frac{1}{\Delta t}+\lambda\right)>\frac{\lambda}{\Lambda} \beta_{k}^{\text {eff }}
$$

If $\Delta t$ is set to infinity, Eq. (44) is required to maintain the inequality of Eq. (43), which makes the exponential transformation unconditionally stable.

$$
\gamma>\frac{\rho(t)}{\Lambda(t)}
$$

If Eq. (44) is not met, the maximum time step can be calculated as:

$$
\Delta t_{\exp }^{\max }=\frac{2}{\left(\frac{\rho-\beta^{e f f}}{\Lambda}-\gamma-\lambda\right)+\sqrt{\left(\frac{\rho-\beta^{e f f}}{\Lambda}-\gamma+\lambda\right)^{2}+4\left(\frac{\lambda}{\Lambda} \beta_{k}^{\text {eff }}\right)}}
$$

By comparing Eq. (45) and Eq. (28), the original $\frac{\rho-\beta^{e f f}}{\Lambda}$ term is reduced by $\gamma$, which is the exponential increase rate predicted with fine time steps. With a good estimate, the $\gamma$ value predicted should be close to the $\frac{\rho-\beta^{e f f}}{\Lambda}$ term which would result in a large increase in the maximum allowable time step.

\section{AMPLITUDE TRANSFORMATION APPLIED TO TRANSPORT EQUATION}

The application of the transformation method to the pin-resolved transport transient equation begins with the factorization of the scalar flux since the isotropic time derivative assumption is made:

$$
\phi(\mathbf{r}, E, t)=T(t) \Phi(\mathbf{r}, E, t)
$$

For a given time step, the PKE is used to calculate the $T(t)$ using multiple $m$ time steps, where the dynamic parameters are linearly interpolated within the time steps. The corresponding solution is $T_{0}, T_{1}, T_{2}, \ldots, T_{m}$, where $T_{0}$ and $T_{m}$ are the amplitude at the beginning and end of the time step, respectively. The number of time steps for the PKEs is the product of the number of CMFD time steps and the number of PKEs time steps performed in the TML second and third levels to maintain the consistency between the transformations and the TML method.

The dynamic parameters at the beginning of the time step are calculated using Eqs. (14) to (17) and based on the flux and material composition at the beginning of the time step. The dynamic parameters at the end of the time step are calculated using Eq. (14) to Eq. (17) and are based on 
the flux at the beginning of the time step but material composition at the end of time step which are estimated using first order perturbation theory [22].

\subsection{Linear Transformation}

Instead of discretizing the scalar flux term $\phi(\mathbf{r}, E, t)$ in Eq. (5), the shape function $\Phi(\mathbf{r}, E, t)$ is discretized using backward Euler:

$$
\frac{\partial \Phi(\mathbf{r}, E, t)}{\partial t}=\frac{\Phi^{n}(\mathbf{r}, E)-\Phi^{n-1}(\mathbf{r}, E)}{\Delta t}
$$

Using the chain rule, the time-dependent equation then becomes:

$$
\begin{aligned}
& \frac{1}{4 \pi v(E)} \frac{\partial \phi(\mathbf{r}, E, t)}{\partial t}=\frac{1}{v(E)}\left(T(t) \frac{\partial \Phi(\mathbf{r}, E, t)}{\partial t}+\Phi(\mathbf{r}, E, t) \frac{\partial T(t)}{\partial t}\right) \\
& =\frac{1}{4 \pi v(E)}\left(\frac{\phi^{n}(\mathbf{r}, E)-\alpha \phi^{n-1}(\mathbf{r}, E)}{\Delta t}+\phi^{n}(\mathbf{r}, E, t) \frac{1-1 / \alpha}{\Delta t}\right)
\end{aligned}
$$

where $\alpha=\frac{T^{n}}{T^{n-1}}$, which is slightly different than the $\alpha$ value in Eq. (37) in order to simplify the equation.

The linear transformation changes the coefficients of Eq. (11), while the other terms stay the same.

$$
\begin{aligned}
& A(\mathbf{r}, E)=-\frac{1}{v(E) \Delta t_{n}}(2-1 / \alpha) \\
& B(\mathbf{r}, E)=\chi_{d}(\mathbf{r}, E)\left(\omega^{n}(\mathbf{r})-\beta(\mathbf{r})\right) \\
& C(\mathbf{r}, E)=\chi_{d}(\mathbf{r}, E) \tilde{S}_{d}^{n-1}(\mathbf{r}, E)+\frac{\alpha \phi^{n-1}(\mathbf{r}, E)}{v(E) \Delta t_{n}}
\end{aligned}
$$

The alpha value can be calculated as $\alpha=\frac{T^{n}}{T^{n-1}}=\frac{T_{m}}{T_{0}}$

\subsection{Exponential transformation}

With the exponential transformation, the amplitude function defined in Eq. (46) follows the exponential behavior defined in Eq. (40) and time derivative term for the transport equation becomes: 


$$
\begin{aligned}
& \frac{1}{4 \pi v(E)} \frac{\partial \phi(\mathbf{r}, E, t)}{\partial t}=\frac{1}{v(E)}\left(T(t) \frac{\partial \Phi(\mathbf{r}, E, t)}{\partial t}+\Phi(\mathbf{r}, E, t) \frac{\partial T(t)}{\partial t}\right) \\
& =\frac{1}{4 \pi v(E)}\left(\frac{\phi^{n}(\mathbf{r}, E)-\phi^{n-1}(\mathbf{r}, E) e^{\gamma \Delta t}}{\Delta t}+\phi^{n}(\mathbf{r}, E) \gamma\right)
\end{aligned}
$$

Accordingly, the transient source terms become:

$$
\begin{aligned}
& A(\mathbf{r}, E)=-\frac{1}{v(E)}\left(\frac{1}{\Delta t_{n}}+\gamma\right) \\
& B(\mathbf{r}, E)=\chi_{d}(\mathbf{r}, E)\left(\omega^{n}(\mathbf{r})-\beta(\mathbf{r})\right) \\
& C(\mathbf{r}, E)=\chi_{d}(\mathbf{r}, E) \tilde{S}_{d}^{n-1}(\mathbf{r}, E)+\frac{e^{\gamma \Delta t} \phi^{n-1}(\mathbf{r}, E)}{v(E) \Delta t_{n}}
\end{aligned}
$$

The exponential increase rate $\gamma$ is calculated using a linear regression technique for the values $\ln T_{0}, \ln T_{1}, \ln T_{2}, \ldots, \ln T_{m}$.

\subsection{Numerical Algorithm}

The overall flow chart for the TML algorithm with the transformation method is shown in Figure 3 , where the three vertical blocks present the three levels of TML solvers. Multiple point kinetics steps are performed prior to the transport predictor time step to calculate the exponential increase rate $\gamma$, and the transformation is then performed. 


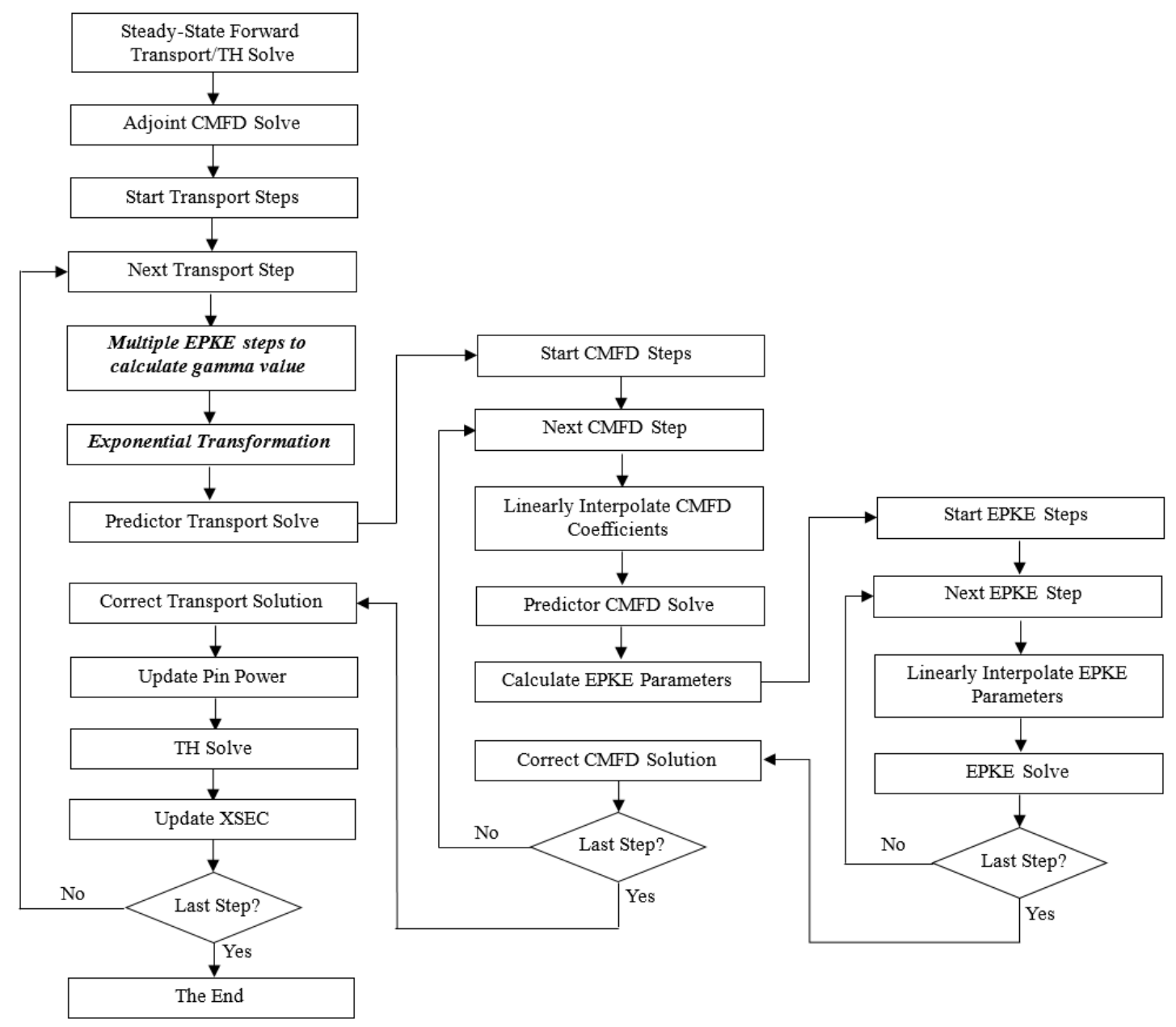

Figure 3 over all flow chart

\section{NUMERICAL RESULTS}

Numerical results are presented in this section in which a single one step transient is performed first in order to examine the maximum time steps predicted by different models for a problem in which the reactivity is inserted at the very beginning of the transient. Three cases are run in this problem, including a pin cell, a 3D SPERT assembly and a 3D SPERT stripe of assemblies. The PKEs predictions and the MPACT results are presented for a standard solver, a linear transformation and an exponential transformation. In the second application, a hot zero power (HZP) SPERT stripe assembly case is used to demonstrate the increase in the maximum time step possible by the exponential transformation method.

\subsection{Accuracy of the PKEs to Predict the Maximum Time Step}

In this section, all the simplest models Eq. (28), Eq. (39) and Eq. (45) are compared results calculated with MPACT. 


\subsubsection{Pin Cell Case}

A typical LWR single pin case (Figure 4) is used first to compare the PKE predictions with MPACT results. The uranium enrichment in the fuel region is varied to introduce different reactivity insertions. The MPACT and PKE predicted maximum time step are shown in Figure 5, and the ratio of the transformed maximum time step and standard maximum time step for linear and exponential transformations are shown in Figure 6 and Figure 7, respectively. The generation time and delay neutron fraction for this pin cell case are 3.6E-5 and 7.12E-3, and the inserted reactivity is fixed at $\$ 1.322$ for both the linear and exponential transformations. Good agreement is observed between the PKEs predictions and MPACT results in these three figures.



Figure 4 Single Pin case geometry

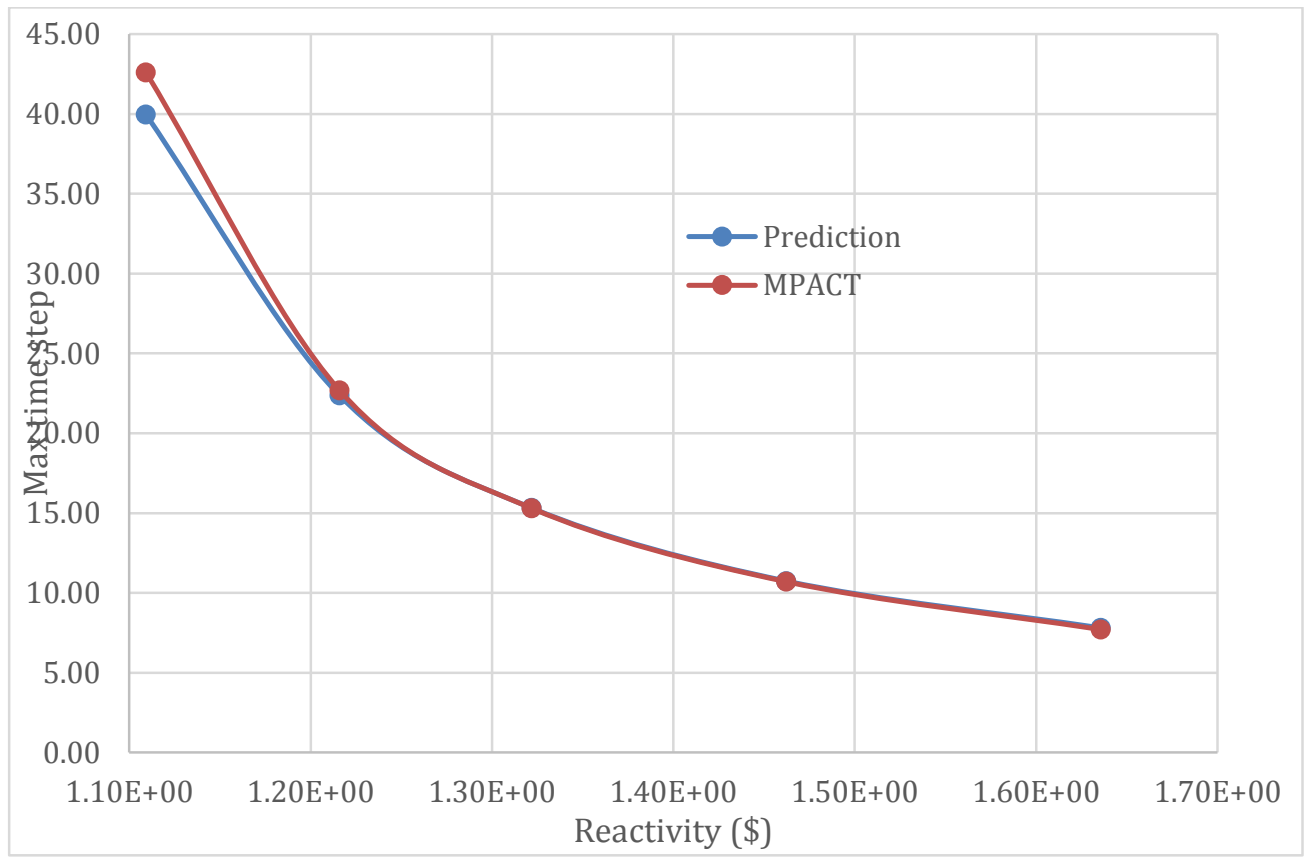

Figure 5 Maximum time step predicted by MPACT and PK model for a pin cell 


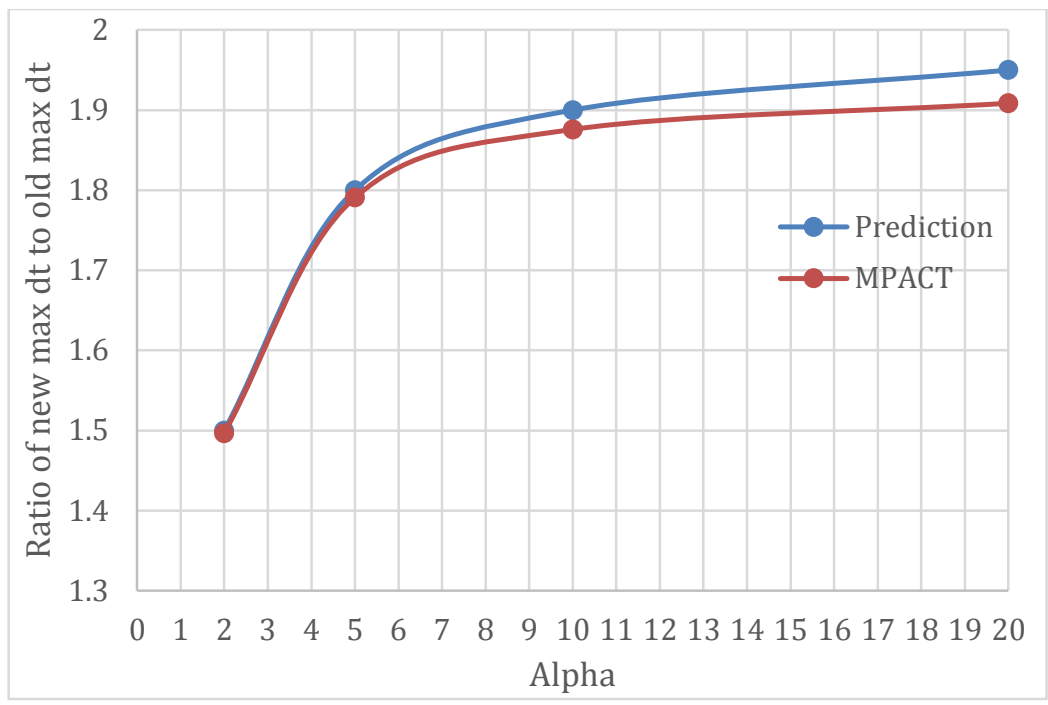

Figure 6 Ratio of new max time step to old max time step using linear transformation for a pin cell

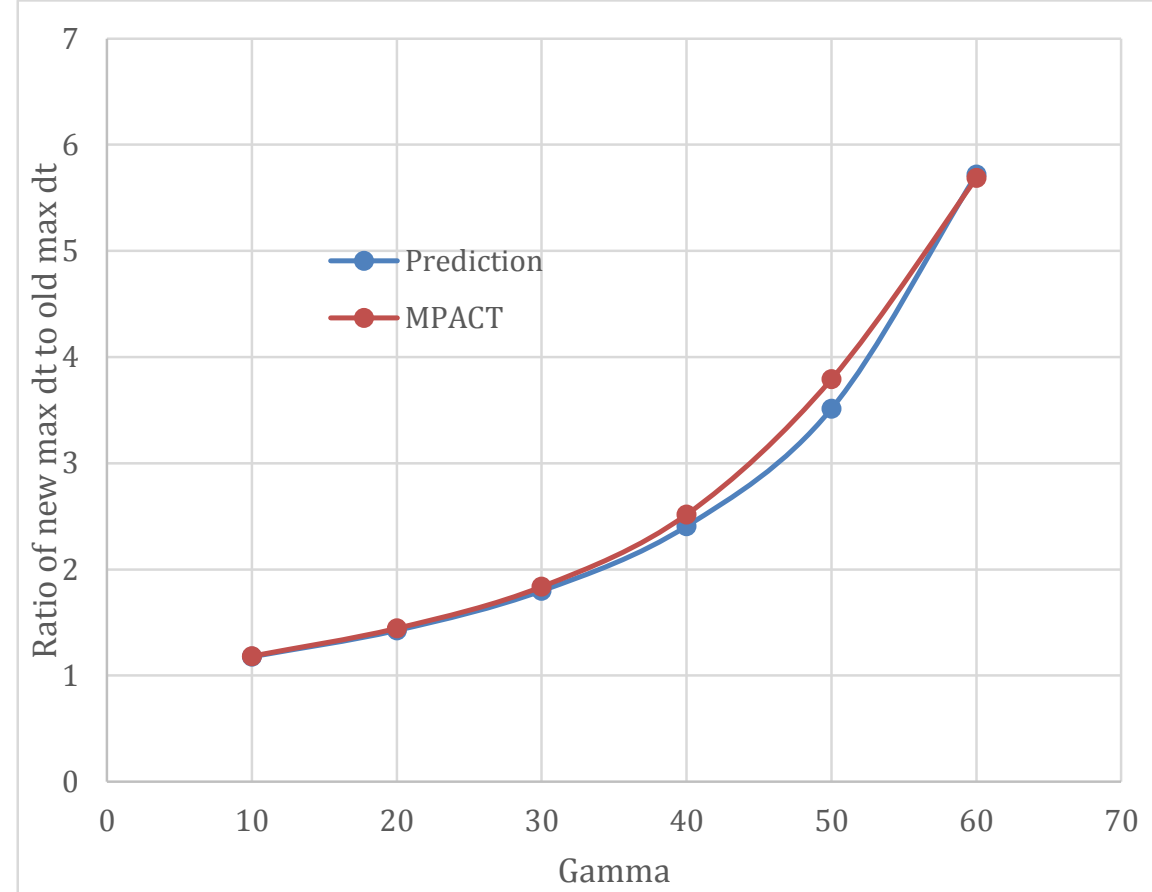

Figure 7 Ratio of new max time step to old max time step using exponential transformation for a pin cell

\subsubsection{D Assembly Case}

The second case used here is a 3D assembly taken from the Special Power Excursion Reactor Test (SPERT), which was established as part of the U. S. Atomic Energy Commission's reactor safety program in 1954. Among the several SPERT core designs, the E-Core consisting of 60 assemblies was used to perform RIA experiments and the data measured during those experiments was used to validate MPACT for application to transient conditions [18]. The 3D 
assembly used here is a 4 by 4 pins array with 20 layers $(120 \mathrm{~cm})$ axially. The radial layout is shown in Figure 8 where the control blade (marked as yellow) is withdrawn. The boron concentration is varied in order to change inserted reactivity.

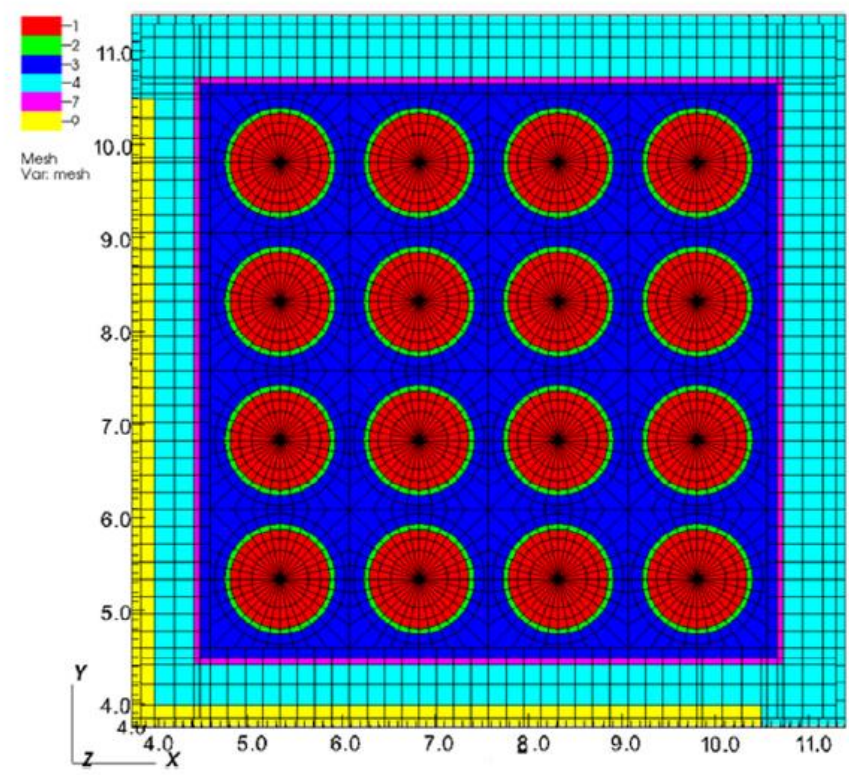

Figure 8 MPACT model of 16-rod assembly with cruciform transient rod

The MPACT and PKE predicted maximum time step are shown in Figure 9 and the ratio of the transformed maximum time step and standard time step for linear and exponential transformation are shown in Figure 10 and Figure 11, respectively. The generation time and delay neutron fraction for this $3 \mathrm{D}$ assembly case are $2.23 \mathrm{E}-5$ and $7.23 \mathrm{E}-3$, and the inserted reactivity is fixed at $\$ 1.318$ for both the linear and exponential transformation. As shown in the Figures, good agreement is observed between the PKE models and the MPACT predictions. 


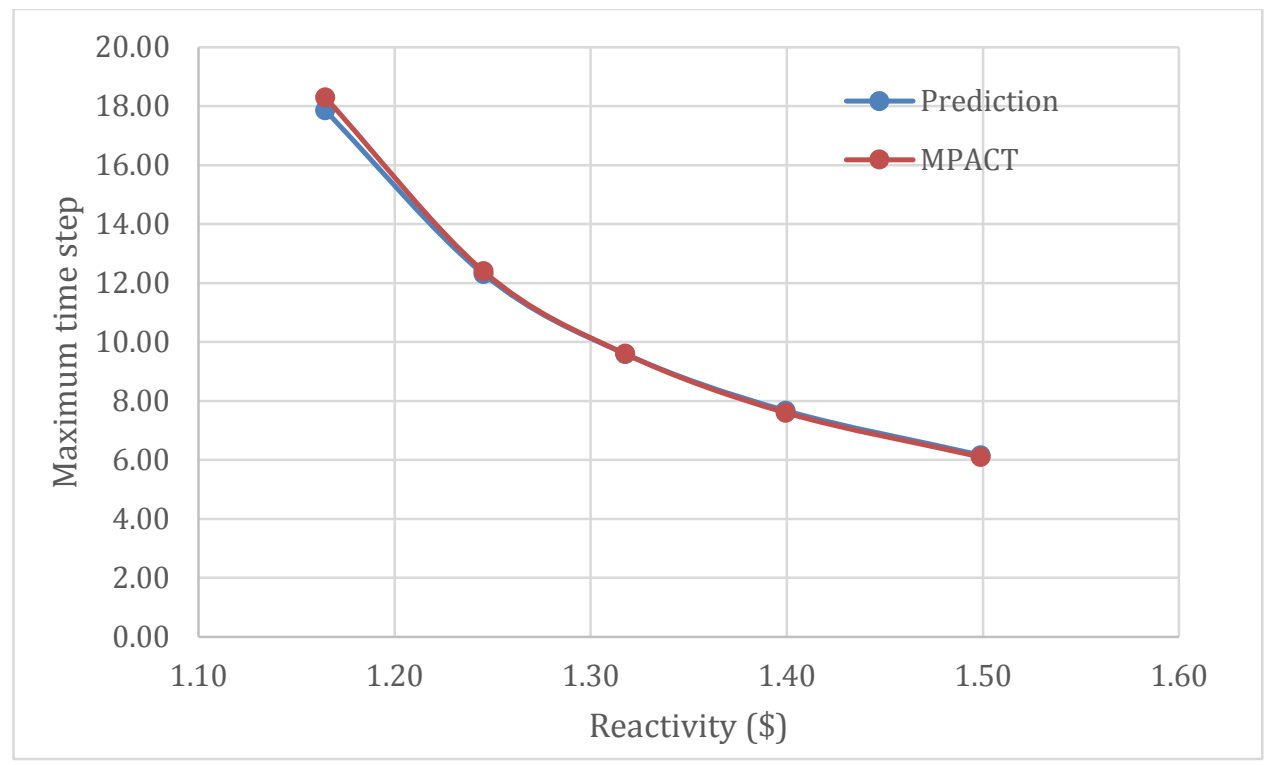

Figure 9 Maximum time step predicted by MPACT and PK model for a 3D assembly

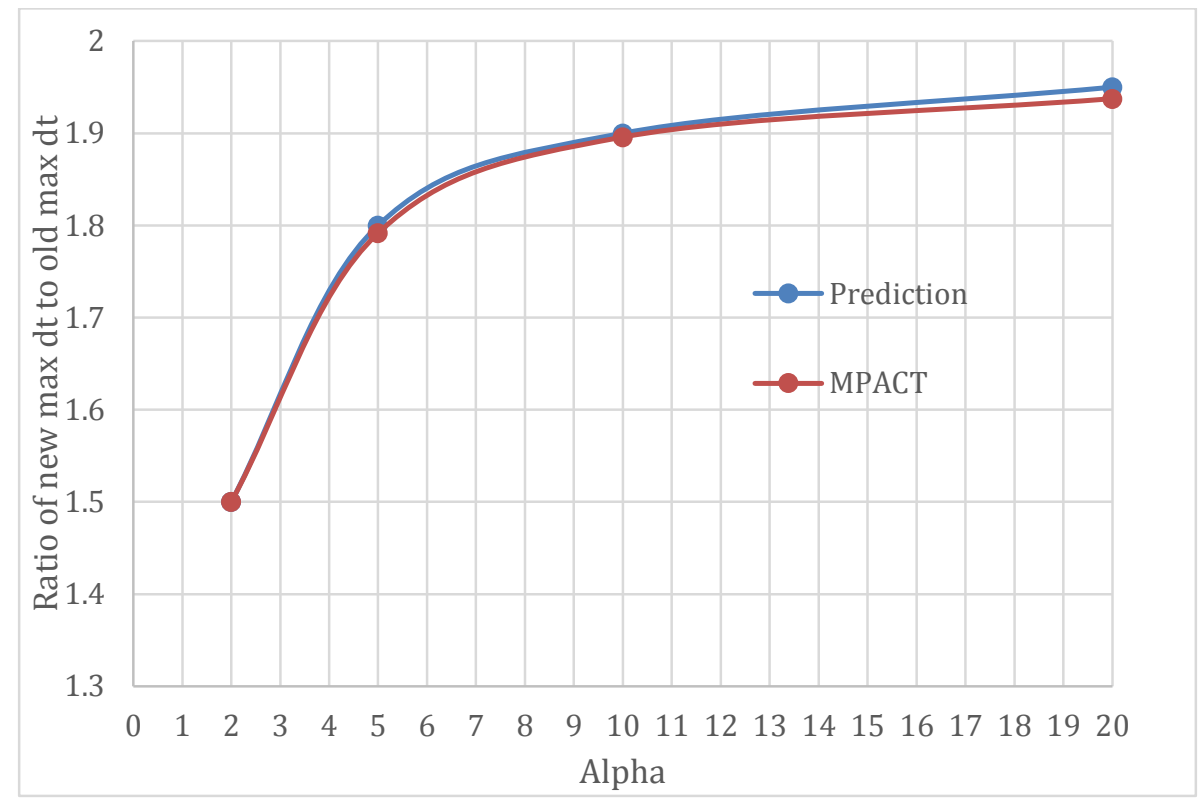

Figure 10 Ratio of new max time step to old max time step using linear transformation for an assembly 




Figure 11 Ratio of new max time step to old max time step using exponential transformation for an assembly

\subsubsection{D "Stripe" Row of Assemblies}

To reduce the computational time and to maintain a problem consistent with practical reactor applications, a single 3D "stripe" row of assemblies in the middle of the SPERT core was developed. The core geometry is shown in Figure 12 and the SPERT III E-core reactor component design data from [23] and [24] are documented in [17]. The MPACT model of the middle of the active core is illustrated in Figure 12 and the stripe assembly was extracted from the first horizontal stripe above the right middle of the core. A vacuum boundary condition is applied to the east, top and bottom of the test problem and a reflective boundary condition is applied for the south, west and north surfaces in the 3D model. 

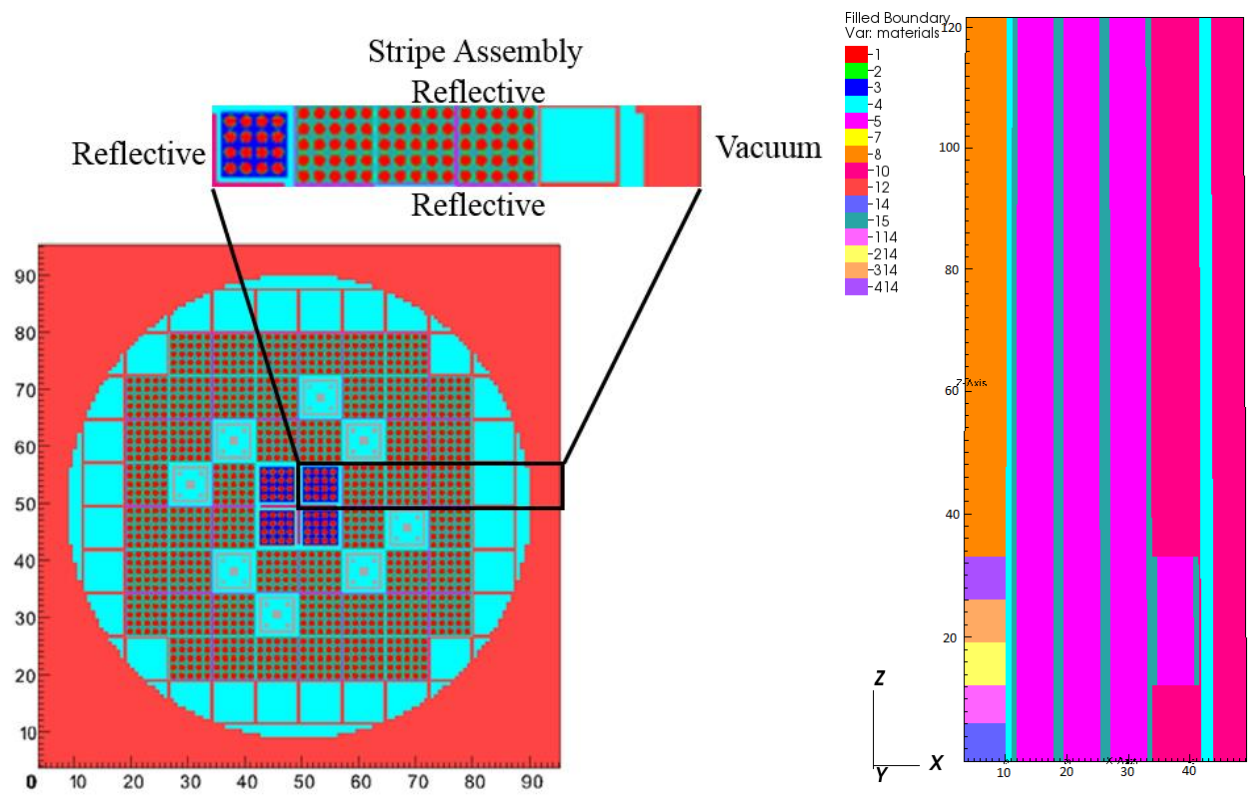

Figure 12 MPACT model for middle of active core and test stripe assembly (left) and MAPCT axial model (right)

The MPACT and point kinetics predicted maximum time steps are shown in Figure 13, and the ratio of the transformed maximum time step and the standard time step for both linear and exponential transformations are shown in Figure 14 and Figure 15, respectively. The generation time and delayed neutron fraction for this $3 \mathrm{D}$ assembly case are $1.64 \mathrm{E}-5$ and $7.43 \mathrm{E}-3$, and the inserted reactivity is fixed at $\$ 1.222$ for both the linear and exponential transformation. As shown in the Figures, good agreement was observed with the PKEs models and MPACT prediction. 


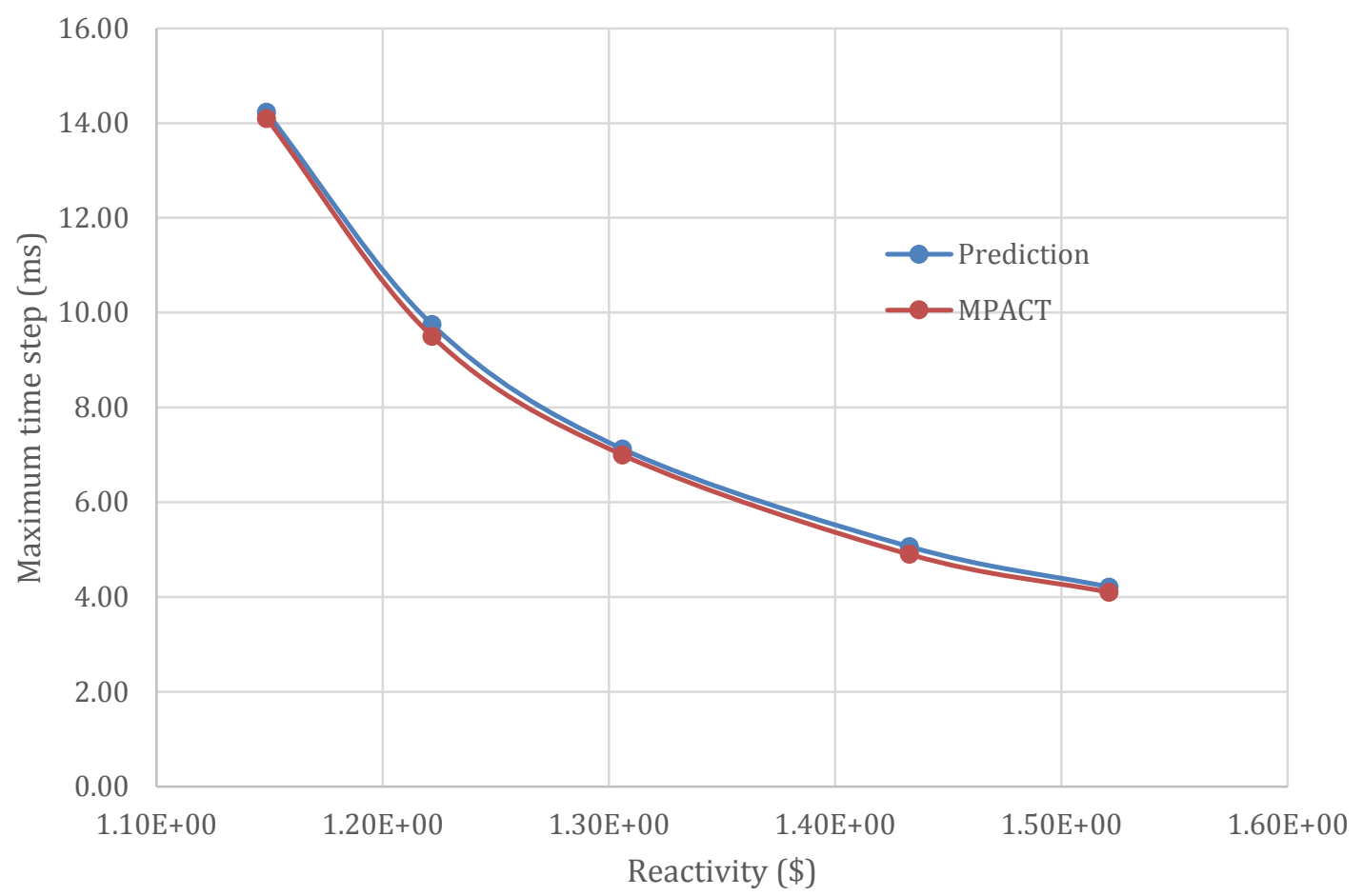

Figure 13 Maximum time step predicted by MPACT and PK model for a stripe assembly

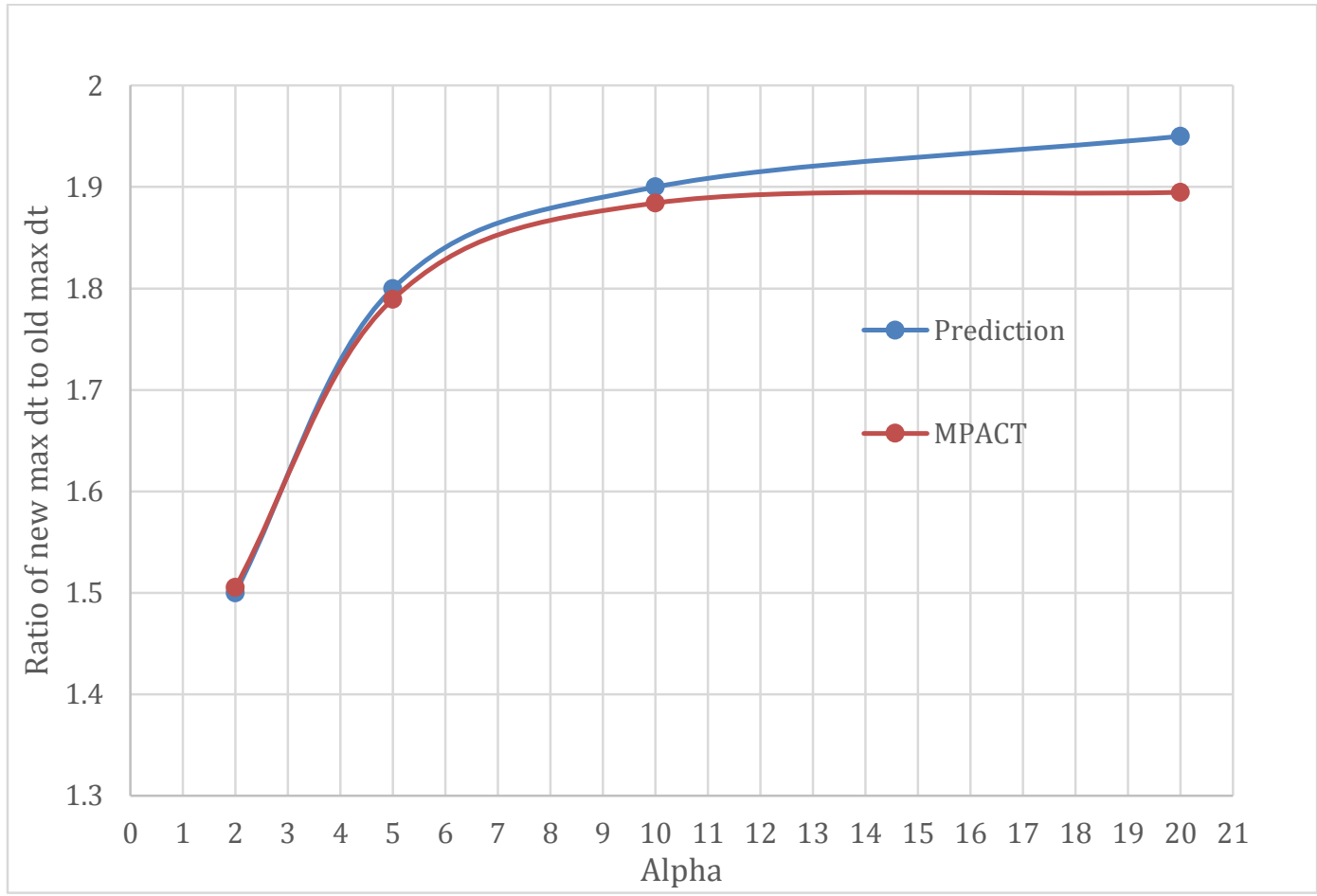

Figure 14 Ratio of new max time step to old max time step using linear transformation for a stripe assembly 


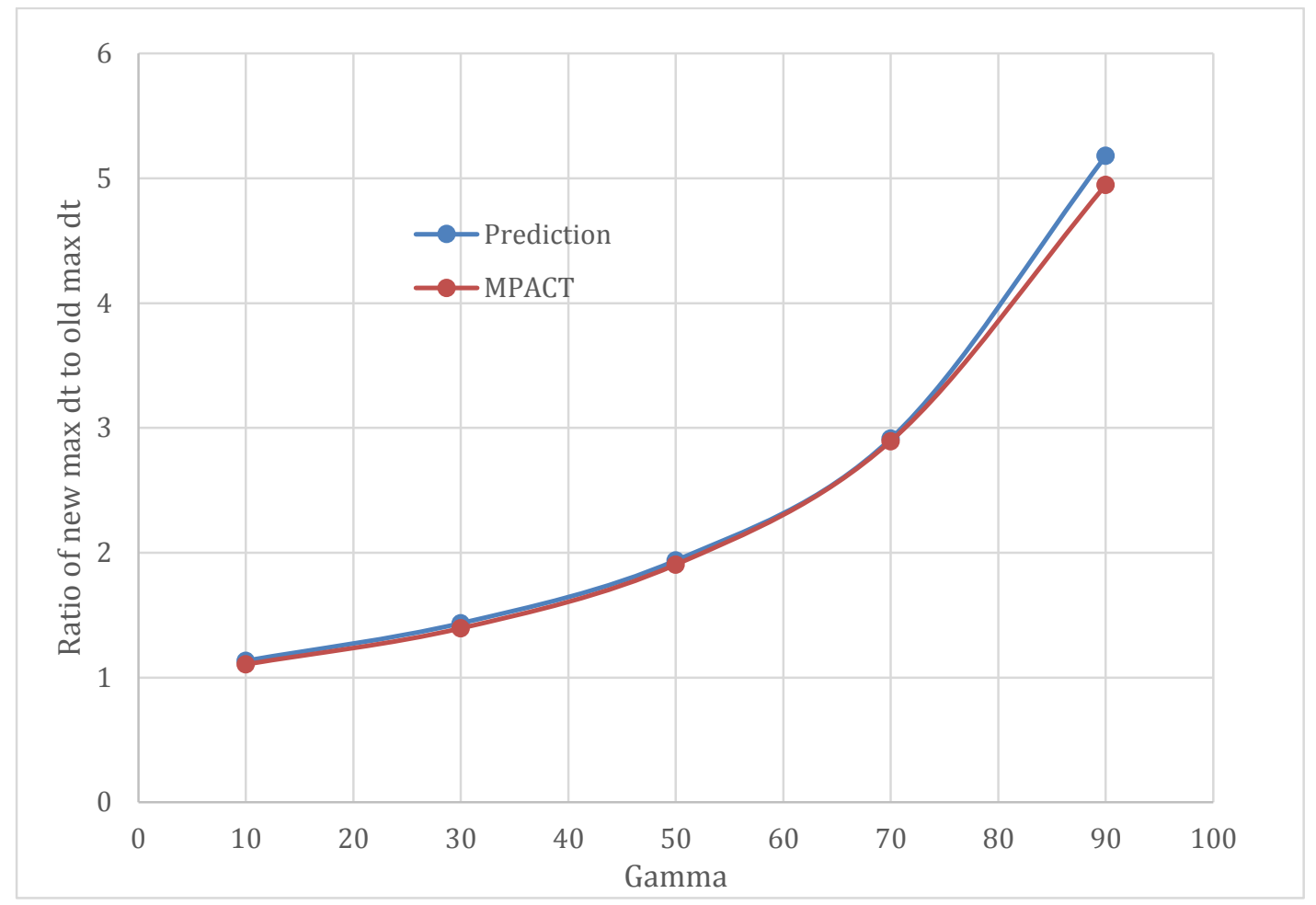

Figure 15 Ratio of new max time step to old max time step using exponential transformation for a stripe assembly

From the three different cases, the PKEs model predictions are sufficiently accurate to predict the maximum time step for all standard, linear and exponential transformations. As predicted in previous section, the linear transformation has less than a factor of 2 increase for the maximum time step, whereas and the exponential transformation method has a much higher increase for the maximum time step, which brings the realistic transient calculation outside the instable region.

\subsection{Whole transient with the striped row assembly.}

The SPERT III E-Core control rod ejection tests consisted of a sequence of CZP (Cold Zero Power), HZP (Hot Zero Power), and HFP (Hot Full Power) tests with various transient rod insertion depths. Among them, test 60 is the most difficult case to simulate due to the significant flux changes which increases from $50 \mathrm{~W}$ to more than $400 \mathrm{MW}$ within 0.3 second. In the reduced model used here, the initial reactor power was set to be $2.5 \mathrm{~W}$ and the inlet temperature was set to $260{ }^{\circ} \mathrm{C}$. The transient blade shown in the left and bottom region in Figure 8 is linearly withdrawn out of the core within $0.1 \mathrm{~s}$ second from the bottom and reaches its maximum dynamic rod worth of $\$ 1.22$.

The SPERT cases were performed in MPACT using 56 energy group cross sections with TCP0 (transport corrected $\mathrm{P} 0$ ) scattering. MOC was performed with $0.05 \mathrm{~cm}$ ray spacing and the Chebyshev-Gauss quadrature set using 4 azimuthal and 1 polar angles. The multi-group NEM 
kernel was used to perform the axial solution of the 2D-1D method in MPACT with 20 axial layers.

The results of six cases with the parameters indicated are shown in Table 2, where Case 1 to Case 3 used standard method and the Case 4 to Case 6 used the exponential transformation methd by varying the MOC time step. The reference solution Case 1 was generated using MOC time steps of $1.0 \mathrm{~ms}$ with 5 CMFD time steps per MOC time step and required 5 hours 10mins execution time on 120 cores on Titan. Case 2 is the three level TML result with 5ms as MOC time step, $1 \mathrm{~ms}$ as CMFD time step and $0.2 \mathrm{~ms}$ as PKEs time step, and the alpha, gamma value predicted are shown in Figure 16. The dynamic reactivity shown in Figure 17 indicates a large reactivity insertion during $0.1 \mathrm{~s}$ to $0.2 \mathrm{~s}$ second after the rod ejection before the $\mathrm{TH}$ feedback playing an important role. Based on Eq. (28), Eq. (39) and Eq. (45), the direct, linear and exponential transformed maximum time step for $0.1 \mathrm{~s}$ to $0.2 \mathrm{~s}$ are plotted in Figure 18. As illustrated, the standard maximum time step is around $10 \mathrm{~ms}$, the linear transformed maximum time step is about $15 \mathrm{~ms}$ and the exponential transformed maximum time step is about $80 \mathrm{~ms}$, which represents a factor of 8 increment for the maximum time step.

Table 2 Cases Performed in this paper

\begin{tabular}{|c|c|c|c|}
\hline Case ID & Transport step size & Transformation & Description \\
\hline 1(Ref.) & $1 \mathrm{~ms}$ & No & $\begin{array}{l}\text { In each transport step, } 5 \text { CMFD time } \\
\text { steps. }\end{array}$ \\
\hline 2 & $5 \mathrm{~ms}$ & No & $\begin{array}{l}\text { In each transport step, } 5 \text { CMFD time } \\
\text { steps; in each CMFD time step, } 5 \text { PK } \\
\text { time steps }\end{array}$ \\
\hline 3 & $5 \mathrm{~ms}$ & No & Pure transport solve \\
\hline 4 & $5 \mathrm{~ms}$ & Exponential & Pure transport solve \\
\hline 5 & $10 \mathrm{~ms}$ & exponential & $\begin{array}{l}\text { In each transport step, } 5 \text { CMFD time } \\
\text { steps; in each CMFD time step, } 10 \\
\text { PK time steps }\end{array}$ \\
\hline 6 & $20 \mathrm{~ms}$ & exponential & $\begin{array}{l}\text { In each transport step, } 10 \text { CMFD time } \\
\text { steps; in each CMFD time step, } 10 \\
\text { PK time steps }\end{array}$ \\
\hline
\end{tabular}



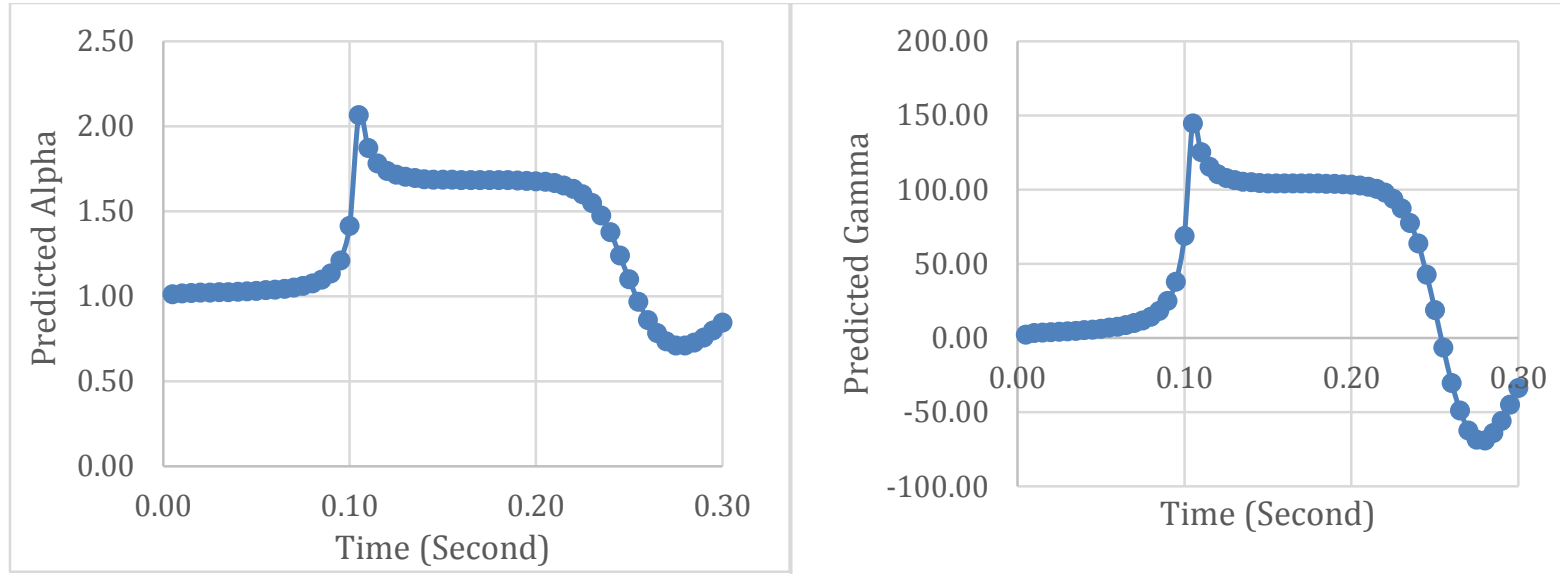

Figure 16 Predicted alpha (left) and predicted gamma (right)

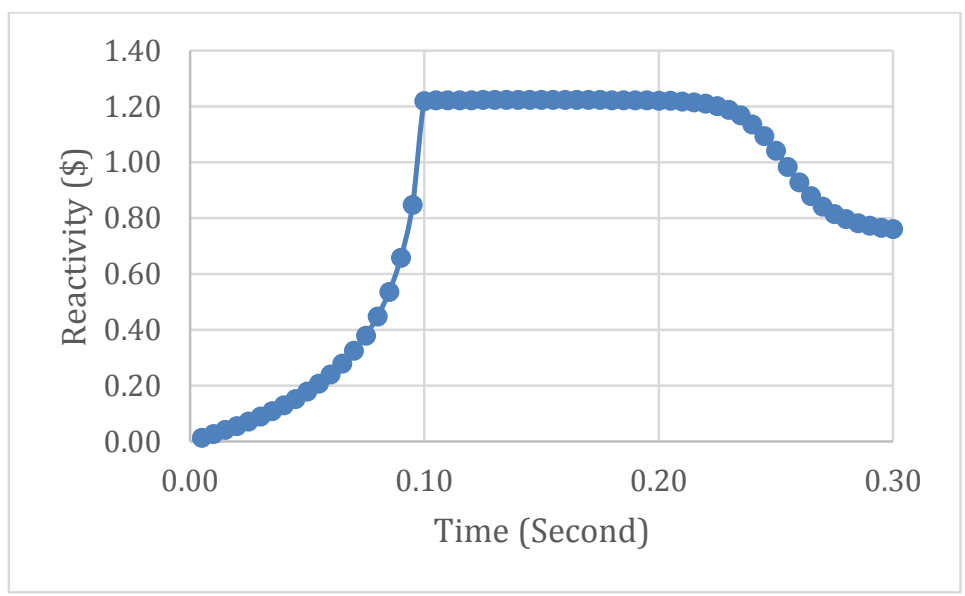

Figure 17 Reactivity as a function of time 


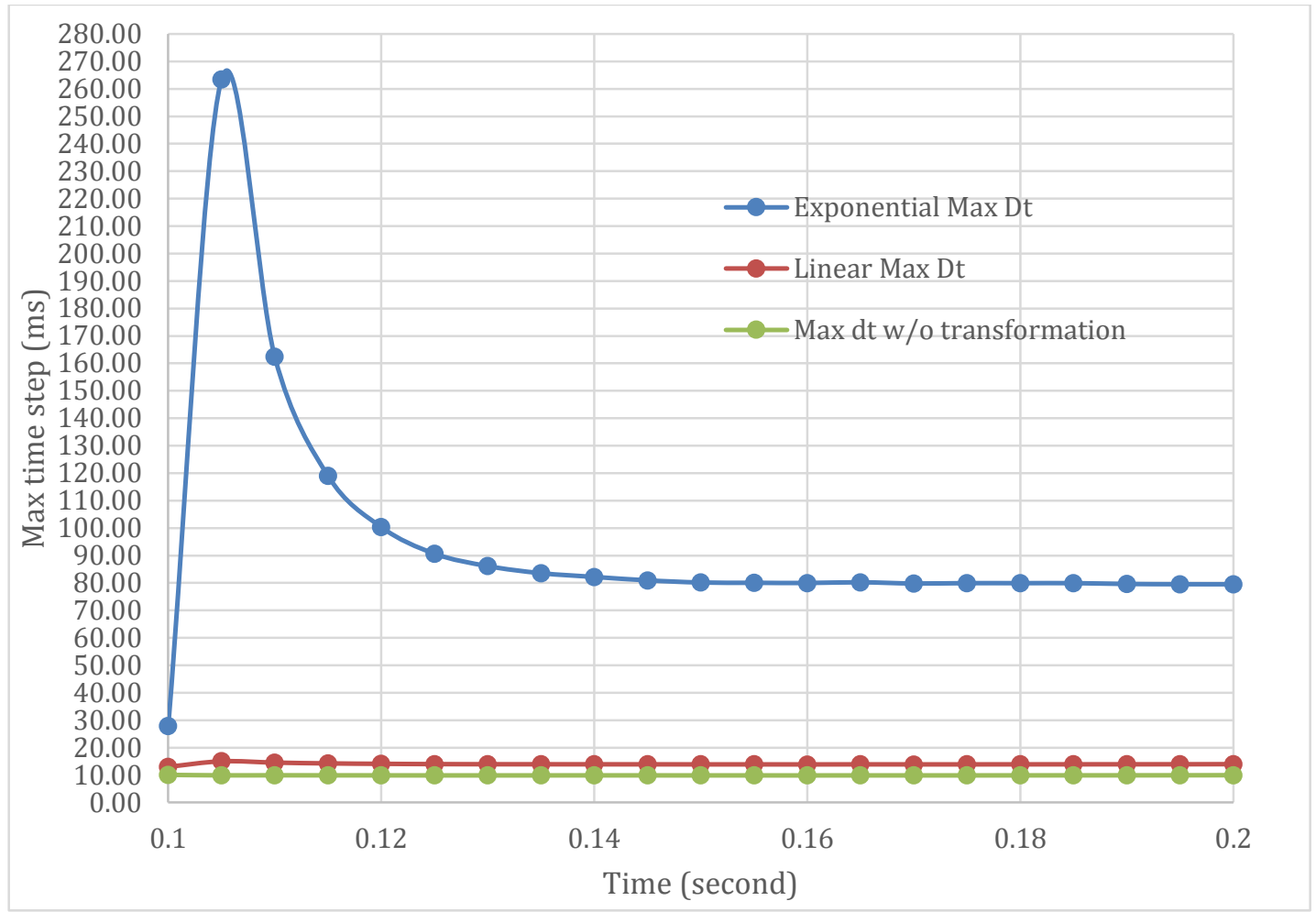

Figure 18 Standard, linear and exponential transformed maximum time step

The power pulse histories for Case 1 to Case 6 are shown in Figure 19 and the relative error for Case 2 to Case 6 are plotted in Figure 20. The relative error shown in Figure 20 is quantified in Eq. (52), and is tabulated in Table 3 as well as the execution time. 


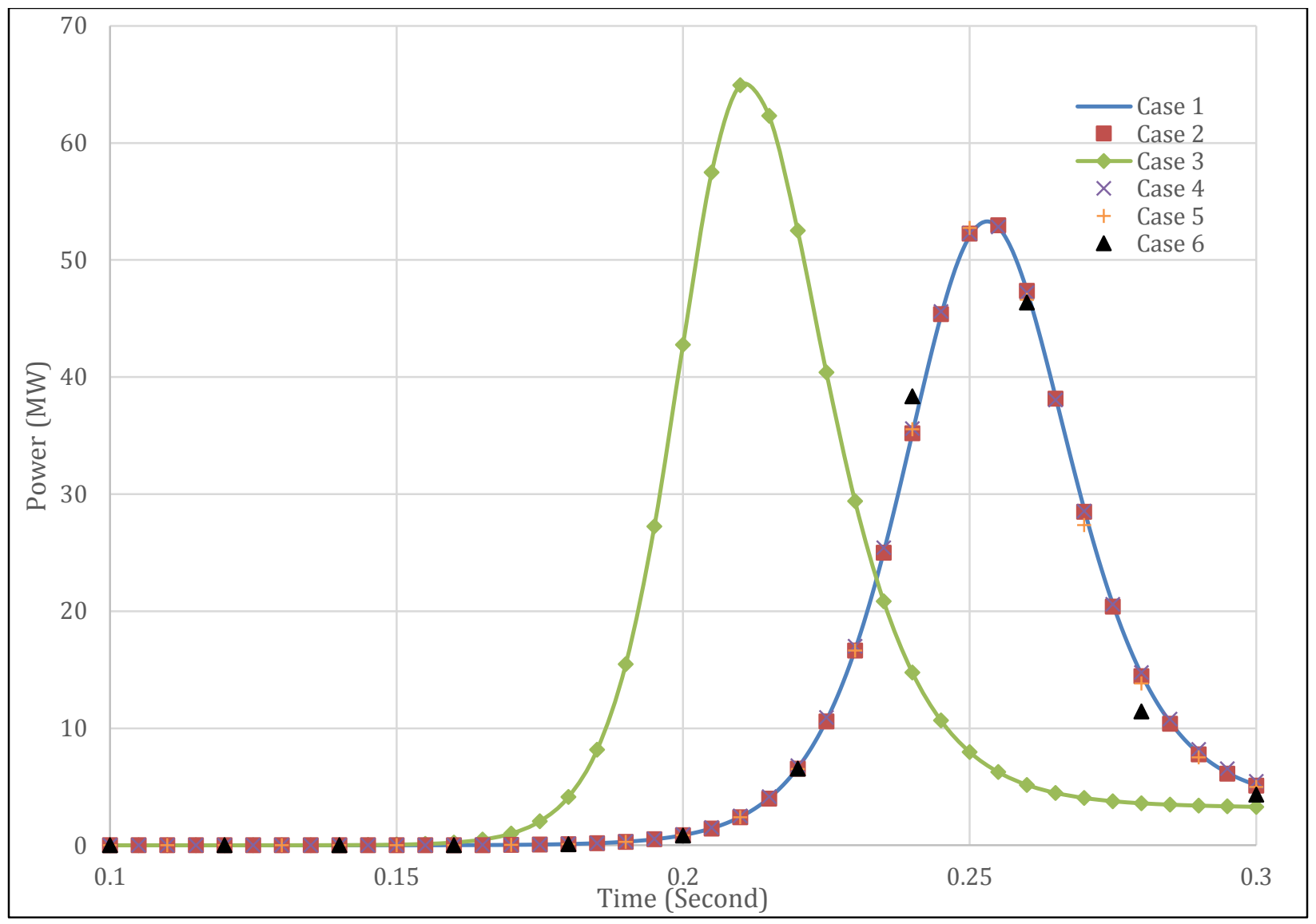

Figure 19 power pulse for stripe assembly

$$
\varepsilon=\sqrt{\frac{1}{N} \sum_{i=1}^{N}\left(\frac{p\left(t_{i}\right)}{p_{r e f}\left(t_{i}\right)}-1\right)^{2}}
$$




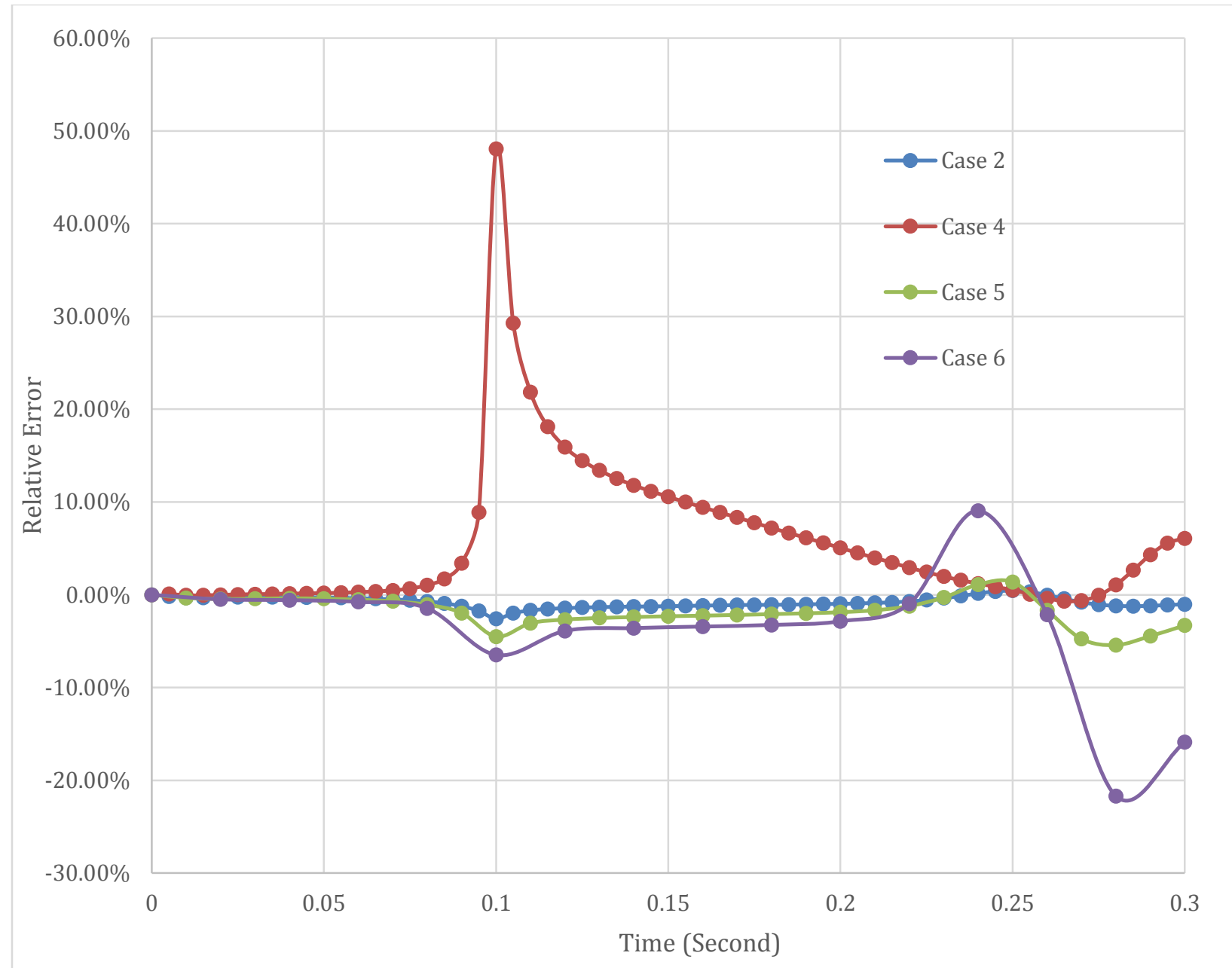

Figure 20 Relative error for stripe assembly

Table 3 Running time and relative error for 6 cases.

\begin{tabular}{lll}
\hline Case ID & Running time & $\begin{array}{l}\text { Average Relative } \\
\text { Error (\%) }\end{array}$ \\
\hline $\mathbf{1}$ & 5 hrs 10mins & - \\
$\mathbf{2}$ & $1 \mathrm{hr} 24 \mathrm{mins}$ & 1.00 \\
$\mathbf{3}$ & $51 \mathrm{mins}$ & 1634.93 \\
$\mathbf{4}$ & $59 \mathrm{mins}$ & 10.00 \\
$\mathbf{5}$ & $47 \mathrm{mins}$ & 2.42 \\
$\mathbf{6}$ & $38 \mathrm{mins}$ & 7.81 \\
\hline
\end{tabular}


By comparing the results of Case 2 and Case 3 to the reference solution in Case 1, the multilevel solver method is essential to capture the power change while maintaining the computational running time. Without exponential transformation, the $10 \mathrm{~ms}$ and $20 \mathrm{~ms}$ standard MOC time step generates negative flux/precursor number density and the success of Case 5 and Case 6 with the exponential transformation demonstrate the capability of performing large time step with exponential transformation. With a larger time step from Case 2 (Case 2 with exponential transformation generates very similar result with Case 4 since the final solution is corrected by TML method), Case 5 to Case 6, a decrement of the accuracy is observed even with TML method due to the fact that the TH coupling is in the MOC time step, where the $20 \mathrm{~ms}$ is too coarse to update temperature feedback. With current $\mathrm{TH}$ feedback iteration scheme, $10 \mathrm{~ms}$ time step is achievable, where the running time is reduced by almost $45 \%$ from $5 \mathrm{~ms}$ time step Case 2 while the accuracy is still acceptable. Finally, the exponential transformation provides better simulation of the core power, which can be demonstrated by comparing Case 3 and Case 4 results with the reference solution Case 1. The overall power pulse in Case 4 overlaps the reference solution in Figure 19, while the direct $5 \mathrm{~ms}$ Case 3 has significant error. However, the Case 4 solution generates large error during the startup of the transient by only using direct exponential transformation shown in Figure 20.

\section{CONCLUSIONS}

The Transient Multi-Level (TML) methodology was developed to improve the computational efficiency of the transient spatial kinetics method by capturing the physical phenomenal in different time domains and thus maximize the numerical accuracy and computational efficiency. One major problem with the TML method was the negative flux/precursor number density generated using large time steps for the MOC solver, which is due to the backward Euler discretization scheme. In this paper, the stability issue of backward Euler discretization was first investigated using the Point Kinetics Equations (PKEs) and the predicted maximum allowed time step for SPERT test 60 case was shown to be less than $10 \mathrm{~ms}$.

To overcome this difficulty, linear and exponential transformations were then investigated using the PKEs. The linear transformation was shown to increase the maximum time step by a factor of

2 , and the exponential transformation is shown to increase the maximum time step by a factor of 5 , as well as provide unconditionally stability above a specified threshold. The two sets of transformations were then applied to TML scheme in the MPACT code, and the numerical results presented show good agreement for standard, linear transformed, and exponential transformed maximum time step between the EPKE model and the MPACT whole core transport solution. Example problems included three different cases, including a pin cell case, a 3D SPERT assembly case and a row of assemblies ("striped assembly case") from the SPERT model. Finally, the successful whole transient execution of the striped assembly case showed the ability 
of the exponential transformation method to use $10 \mathrm{~ms}$ and $20 \mathrm{~ms}$ time steps, which all failed using the standard method.

\section{ACKNOWLEDGMENTS}

This research was supported by the Consortium for Advanced Simulation of Light Water Reactors (www.casl.gov), an Energy Innovation Hub (http:/www.energy.gov/hubs) for Modeling and Simulation of Nuclear Reactors under U.S. Department of Energy Contract No. DE-AC05$00 \mathrm{OR} 22725$.

\section{REFERENCES}

1. Henry, A.F., 1958. The application of reactor kinetics to the analysis of experiments. Nuclear Science and Engineering 3, 52-70.

2. Henry, A.F., Curlee, N.J., 1958. Verification of a method for treating neutron space-time problems. Nuclear Science and Engineering 4, 727-744.

3. Ott, K.O., Meneley, D.A., 1969. Accuracy of the quasistatic treatment of spatial reactor kinetics. Nuclear Science and Engineering 36, 402-411.

4. Ban Y, Endo T, Yamamoto A. A unified approach for numerical calculation of spacedependent kinetic equation[J]. Journal of nuclear science and technology, 2012, 49(5): 496515.

5. Kosuke Tsujita, Tomohiro Endo, and Akio Yamamoto. "Application of the multigrid amplitude function method for time-dependent transport equation using moc." In: Proc. Mathematics and Computational Methods Applied to Nuclear Science \& Engineering. M\&C 2013. Sun Valley, ID, May 5-9, 2013 (2013).

6. Samuel Shaner, Benoit Forget, and Kord Smith. "Sensitivity Analysis and Performance of the Adiabatic,Theta, and Multigrid Amplitude Function Kinetics Methods in 2D MOC Neutron Transport." In: Proc. Mathematics and Computational Methods Applied to Nuclear Science \& Engineering. M\&C 2013. Sun Valley, ID, May 5-9, 2013 (2013).

7. Kao, P.W., Henry, A.F., 1989. Supernodal analysis of PWR transients. In: Hall, M. (Ed.), Proceedings of the Topical Meeting on Advances in Nuclear Engineering Computation and Radiation Shielding, American Nuclear Society, Santa Fe, New Mexico, 9-13 April 1989, p. 63

8. Sandra Dulla, etc. "Accuracy of a Predictor-Corrector Quasi-Static Method for Space-Time Reactor Dynamics.” PHYSOR 2006. Vancouver, BC, Sept 10-14, 2006 (2006).

9. Dulla, S., Mund, E.H., Ravetto, P., 2008. The quasi-static method revisited. Prog. Nucl. Energy 50, 908-920. 
10. Caron D, Dulla S, Ravetto P. New aspects in the implementation of the quasi-static method for the solution of neutron diffusion problems in the framework of a nodal method[J]. Annals of Nuclear Energy, 2015.

11. Ang Zhu, et all, “A Multi-level Quasi-Static Kinetics Method for Pin-Resolved Transport Transient Reactor Analysis”, Nuclear Science and Engineering, Accepted.

12. Wang R C, Xu Y, Hudson N, et al. Validation of the US NRC Coupled Code System TRITON/TRACE/PARCS Using the Special Power Excursion Reactor Test III[J]. Nuclear Technology, 2013, 183(3): 504-514.

13. Reed W H, Hansen K F. Alternating direction methods for the reactor kinetics equations[J]. Nuclear Science and Engineering, 1970, 41(3): 431-442.

14. Ferguson D R, Hansen K F. Solution of the space-dependent reactor kinetics equations in three dimensions[J]. Nuclear Science and Engineering, 1973, 51(2): 189-205.

15. Buckner M R, Stewart J W. Multidimensional Space-Time Nuclear-Reactor Kinetics Studies_-Part I: Theoretical[J]. Nuclear Science and Engineering, 1976, 59(4): 289-297.

16. MPACT Team, MPACT Theory Manual, Version 1.0 , University of Michigan, Ann Arbor, MI, October, 2013

17. L. Cao, et al., "Neutronics Modeling of the SPERT III E-Core Critical Experiments with MPACT and KENO,” Annals of Nuclear Energy, 80 (2015) 207-218

18. A. Zhu, et al, "Transient Methods For Pin-Resolved Whole Core Transport Using The 2d-1d Methodology In Mpact", Joint International Conference on Mathematics and Computation (M\&C), Supercomputing in Nuclear Applications (SNA) and the Monte Carlo (MC) Method (M\&C 2015), American Nuclear Society, Nashville, TN, USA, April 19-23, [CD-ROM] (2015)

19. T. Downar, Y. Xu, V. Seker, PARCS v3.0 U.S. NRC Core Neutronics Simulator User/Theory Manual, Department of Nuclear Engineering and Radiological Sciences University of Michigan, Ann Arbor, MI, September 2009.

20. A. Zhu, et al, "The Implementation and Analysis of the MOC and CMFD Adjoint Capabilities in the 2D-1D Code MPACT”, Joint International Conference on Mathematics and Computation (M\&C), Supercomputing in Nuclear Applications (SNA) and the Monte Carlo (MC) Method (M\&C 2015), American Nuclear Society, Nashville, TN, USA, April 1923, [CD-ROM] (2015)

21. Ang Zhu, Yunlin $\mathrm{Xu}$, and $\mathrm{T}$. Downar, Fourier Convergence Analysis of the Infinite Homogenous Multi-Group Time Dependent Boltzmann Transport Equation Using SN Formulation. Annals of Nulcear Energy, In preparation.

22. Karl O. Ott, Introductory Nuclear Reactor Dynamics, American Nuclear Society (December 1985) 
23. J. Durgone, SPERT III Reactor Facility: E-CORE Revision, AEC Research and Development Report IDO-17036, November 1965.

24. M. Jessee, M.D. DeHART, TRITON: A Multipurpose Transport, Depletion, and Sensitivity and Uncertainty Analysis Module, T1.3.11, Version 6.1, Sect T1, Oak Ridge National Laboratory, Reactor and Nuclear Systems Division, June 2011. 